\title{
Brainstem encoding of speech and musical stimuli in congenital amusia: evidence from Cantonese speakers
}

\author{
Fang Liu', Akshay R. Maggu' ${ }^{1}$, Joseph C. Y. Lau' ${ }^{1}$ and Patrick C. M. Wong 1,2,3,4 \\ ' Department of Linguistics and Modern Languages, The Chinese University of Hong Kong, Hong Kong, China \\ ${ }^{2}$ The Chinese University of Hong Kong - Utrecht University Joint Center for Language, Mind and Brain, Hong Kong, China \\ ${ }^{3}$ Roxelyn and Richard Pepper Department of Communication Sciences and Disorders, Northwestern University, Evanston, IL, USA \\ ${ }^{4}$ Department of Otolaryngology, Head and Neck Surgery, Northwestern University Feinberg School of Medicine, Chicago, IL, USA
}

\section{Edited by:}

Simone Dalla Bella, University of

Montpellier 1, France

\section{Reviewed by:}

Barbara Tillmann, Centre National de la Recherche Scientifique, France Gavin M. Bidelman, University of Memphis, USA

\section{*Correspondence:}

Patrick C. M. Wong, Department of Linguistics and Modern Languages, The Chinese University of Hong Kong, Room G03, Leung Kau Kui Building, Shatin, New Territories, Hong Kong, China

e-mail:p.wong@cuhk.edu.hk
Congenital amusia is a neurodevelopmental disorder of musical processing that also impacts subtle aspects of speech processing. It remains debated at what stage(s) of auditory processing deficits in amusia arise. In this study, we investigated whether amusia originates from impaired subcortical encoding of speech (in quiet and noise) and musical sounds in the brainstem. Fourteen Cantonese-speaking amusics and 14 matched controls passively listened to six Cantonese lexical tones in quiet, two Cantonese tones in noise (signal-to-noise ratios at 0 and $20 \mathrm{~dB}$ ), and two cello tones in quiet while their frequency-following responses (FFRs) to these tones were recorded. All participants also completed a behavioral lexical tone identification task. The results indicated normal brainstem encoding of pitch in speech (in quiet and noise) and musical stimuli in amusics relative to controls, as measured by FFR pitch strength, pitch error, and stimulus-toresponse correlation. There was also no group difference in neural conduction time or FFR amplitudes. Both groups demonstrated better FFRs to speech (in quiet and noise) than to musical stimuli. However, a significant group difference was observed for tone identification, with amusics showing significantly lower accuracy than controls. Analysis of the tone confusion matrices suggested that amusics were more likely than controls to confuse between tones that shared similar acoustic features. Interestingly, this deficit in lexical tone identification was not coupled with brainstem abnormality for either speech or musical stimuli. Together, our results suggest that the amusic brainstem is not functioning abnormally, although higher-order linguistic pitch processing is impaired in amusia. This finding has significant implications for theories of central auditory processing, requiring further investigations into how different stages of auditory processing interact in the human brain.

Keywords: congenital amusia, brainstem, pitch, lexical/musical tone, speech in noise, Cantonese, frequencyfollowing response (FFR)

\section{INTRODUCTION}

Congenital amusia is a neuro-genetic disorder of musical processing (Drayna et al., 2001; Peretz et al., 2007), affecting around $4 \%$ of the general population for both tone and non-tonal language speakers (Kalmus and Fry, 1980; Nan et al., 2010; Wong et al., 2012; although see Henry and McAuley, 2010, 2013, for criticisms). Impacting basic music production and perception abilities (Ayotte et al., 2002), this disorder is also associated with impaired fine-grained pitch discrimination (Peretz et al., 2002; Jiang et al., 2011), elevated thresholds for pitch change detection and pitch direction identification/discrimination (Foxton et al., 2004; Hyde and Peretz, 2004; Liu et al., 2010, 2012b; Jiang et al., 2013), and impaired short-term memory for pitch (Gosselin et al., 2009; Tillmann et al., 2009; Williamson and Stewart, 2010; Albouy et al., 2013a).

Although some early studies reported ceiling performance of amusics on speech intonation processing, presumably due to the coarse intonational contrasts used (Ayotte et al., 2002; Peretz et al., 2002; Patel et al., 2005), more recent research has suggested that amusia is a domain-general pitch processing deficit that also compromises subtle aspects of pitch processing in speech, including lexical tone perception, linguistic and emotional prosody processing, and speech intonation imitation (Patel et al., 2008; Hutchins et al., 2010; Jiang et al., 2010, 2012a,b; Liu et al., 2010, 2012a, 2013; Nan et al., 2010; Tillmann et al., 2011a,b; Thompson et al., 2012). The non-modularity of pitch deficits in amusia has recently been confirmed by a quantitative review through meta-analysis of the previous studies (Vuvan et al., 2014). Furthermore, amusics also show impaired time matching abilities in speech imitation (Liu et al., 2013), and demonstrate reduced speech comprehension in both quiet and noise, with either natural or flattened pitch contours (Liu et al., 2015).

Structural neuroimaging studies suggest that the amusic brain differs from neurotypical brains in subtle ways. For example, the amusic brain has reduced white matter and increased gray matter in the right inferior frontal gyrus (Hyde et al., 2006; Albouy et al., 2013a), reduced gray matter in the right superior temporal gyrus (Albouy et al., 2013a), and thicker cortex in the right 
inferior frontal gyrus and the right superior temporal gyrus (Hyde et al., 2007). Furthermore, amusics also show reduced gray matter in the left inferior frontal gyrus and the left superior temporal sulcus (Mandell et al., 2007), and reduced arcuate fasciculus connectivity along the right frontotemporal pathway (Loui et al., 2009).

Despite anatomical abnormalities of the amusic brain, most event-related potentials (ERPs) studies have revealed near-normal early pre-attentive brain potentials of amusics (e.g., N100, N200, MMN, P200) in response to small pitch changes and musical incongruities/violations (Peretz et al., 2005, 2009; Moreau et al., 2009, 2013; Mignault Goulet et al., 2012; Omigie et al., 2013), which they often fail to detect at the behavioral level, nor do they respond through late brain potentials that represent attentive processing (e.g., P300, P600; Peretz et al., 2009; Jiang et al., 2012a; Mignault Goulet etal., 2012; Moreau et al., 2013). Furthermore, in an functional magnetic resonance imaging (fMRI) study, amusics demonstrated normal brain activities in both the left and right auditory cortices when passively listening to puretone sequences with varying pitch distances, although abnormal deactivation in the right inferior frontal gyrus, decreased connectivity along the right frontotemporal pathway, and abnormal over-connectivity between the left and right auditory cortices were also observed (Hyde et al., 2011). Together, these findings suggest that amusics lack the ability to process subtle pitch variations and music structure consciously, albeit they can do so pre-attentively. Therefore, it seems that the amusic deficits are outside of the auditory cortex, which is assumed to generate early brain potentials, but instead reside in the right inferior frontal gyrus and the right frontotemporal pathway (Hyde et al., 2011; Peretz, 2013).

Nevertheless, a recent magnetoencephalography (MEG) study revealed a decreased and delayed N100m in bilateral inferior frontal gyrus and Heschl's gyrus/superior temporal gyrus of the amusic brain during a melodic contour discrimination task, suggesting that pitch processing deficits in amusia might start from the auditory cortex (Albouy et al., 2013a). This is consistent with a few other ERP studies which have also observed abnormal early brain responses (e.g., N100, MMN) in amusia (Braun et al., 2008; Jiang et al., 2012a; Omigie et al., 2013). Thus, it remains to be determined at what stage(s) of auditory processing deficits in amusia arise.

At the behavioral level, amusics also demonstrate preserved pitch and music processing abilities in an implicit manner, but not explicitly like typical listeners do. For example, amusics were better able to imitate than identify/discriminate pitch direction and speech intonation, presumably because of unconscious pitch processing during imitation (Loui et al., 2008; Liu et al., 2010; Hutchins and Peretz, 2012). In a melodic discrimination task, amusics showed implicit processing of melodic structure in Western tonal music through faster responses times (but not better performance) to tonal than atonal sequences (Albouy et al., 2013b). They also exhibited implicit processing of melodic expectation (high versus low probability notes) and harmonic structure in priming tasks (Omigie et al., 2012; Tillmann et al., 2012), but were unable to perform as well as controls in the explicit rating task (Omigie et al., 2012). Similarly, although amusics self-reported to be unable to recognize melodies without lyrics, they rated familiarity of instrumental music as well as controls, demonstrating implicit storage of familiar melodies in long-term memory (Tillmann et al., 2014).

The domain-generality and the neural origin(s) of the amusic deficits, together with the intriguing dissociation between pre-attentive/implicit and attentive/explicit processing of pitch in amusia, warrant further investigation. In particular, it is unclear whether the cortical dysfunctions revealed by previous studies were driven by the ascending pathway that started earlier, e.g., the brainstem, and if so, whether the domain-generality of pitch processing also manifests at the brainstem level in amusia. It has been shown that the ability to decode speech/music sounds in a meaningful manner is a complex task involving multiple stages of neural processing (Poeppel and Hickok, 2004; Peretz and Zatorre, 2005; Stewart et al., 2006; Hickok and Poeppel, 2007; Poeppel et al., 2008). Before speech/music sounds can be perceived and mapped onto long-term mental representations in the cortex, relevant acoustic properties such as temporal and spectral information must be represented and transformed through a neural code by subcortical structures, including the auditory nerve, the brainstem, the midbrain, etc. (Eggermont, 2001). It is well established that the auditory brainstem represents elements of speech/music sounds, such as timing, frequency, and timbre, with remarkable fidelity (Chandrasekaran and Kraus, 2010; Skoe and Kraus, 2010). This representation is also influenced by language experience (Krishnan et al., 2005, 2010b,c; Krizman et al., 2012), musicianship (Musacchia et al., 2007; Wong et al., 2007; Parbery-Clark etal., 2009a; Strait etal., 2012), and short-term auditory training (Song et al., 2008; Carcagno and Plack, 2011; Chandrasekaran etal., 2012; Anderson et al., 2013). Given the impact of long-term experience on the brainstem, it is necessary to examine whether amusics' deficits start earlier at the brainstem, rather than being confined within the cortex, for both musical and speech stimuli.

In addition, given that musicians demonstrate enhanced brainstem encoding of speech and music stimuli as well as speech in noise compared to non-musicians (Musacchia et al., 2007; Wong et al., 2007; Parbery-Clark et al., 2009a; Strait et al., 2012), it will be interesting to examine whether amusics would show reduced brainstem encoding of these stimuli relative to controls. The answer to this question would help establish whether subcortical encoding of speech/music sounds reflects musical aptitude along the entire spectrum from musicians to non-musician controls and to amusics.

In order to most comprehensively interrogate whether pitchprocessing deficits in amusia originate from the brainstem, we examined amusics' frequency-following responses (FFRs) to an array of stimuli in the present study, including speech-in-quiet, speech-in-noise, and musical tones. FFR is scalp-recorded electrophysiological response originated from the rostral brainstem, reflecting phase-locked neural activity in the brainstem (Smith et al., 1975; Sohmer et al., 1977). It is synchronized to the temporal structure (and thus periodicity) of the evoking stimulus (Chandrasekaran and Kraus, 2010; Skoe and Kraus, 2010). Thus, FFR has the potential to reveal subtle pitch tracking problems of amusics that cortical ERPs cannot. Apart from FFR recordings, 
we also examined whether there is a dissociation between brainstem representation and behavioral identification of pitch in amusia.

In order to elicit meaningful identification of natural and behaviorally relevant pitches, we used native speakers of Cantonese as participants and Cantonese tones as part of test materials. Cantonese is a tone language with a highly complex tonal system (Yip, 2002). There are six lexical tones in Cantonese, named Tone 1 to Tone 6 . Using the scale of 1-5 (5 being the highest and 1 being the lowest tonal point; Chao, 1930), the high-level Cantonese Tone 1 can be transcribed as having pitch values of 55, the high-rising Tone 2 as having 25, the mid-level Tone 3 as 33, the low-falling Tone 4 as 21, the low-rising Tone 5 as 23, and the low-level Tone 6 as 22 (Figure 1). The fact that there are multiple tones with similar acoustic features in Cantonese (Rising: 25/23; Level: 55/33/22; Falling: 21) makes tone perception challenging even for native speakers (Varley and So, 1995; Ciocca and Lui, 2003; Francis and Ciocca, 2003). It has been found that tone language speakers have enhanced brainstem encoding of native tonal features, e.g., acceleration rates of pitch rises (Krishnan et al., 2005, 2010b,c), presumably because of perceptual learning of native sounds through long-term exposure to the sound environment. Whether amusic tone language speakers would demonstrate such a brainstem-encoding enhancement like typical listeners do is an open question. Given that lexical and non-lexical pitch processing across different tone language speakers (e.g., Chinese versus Thai) differentiate themselves cortically (Gandour et al., 2002), but not subcortically (Krishnan et al., 2010b), examining tone language speakers and lexical tone processing would enable us to determine whether or not there is a dissociation between brainstem representation and behavioral identification of pitch in amusia.

To this end, we conducted a set of FFR experiments to examine whether Cantonese-speaking amusics have deficits in

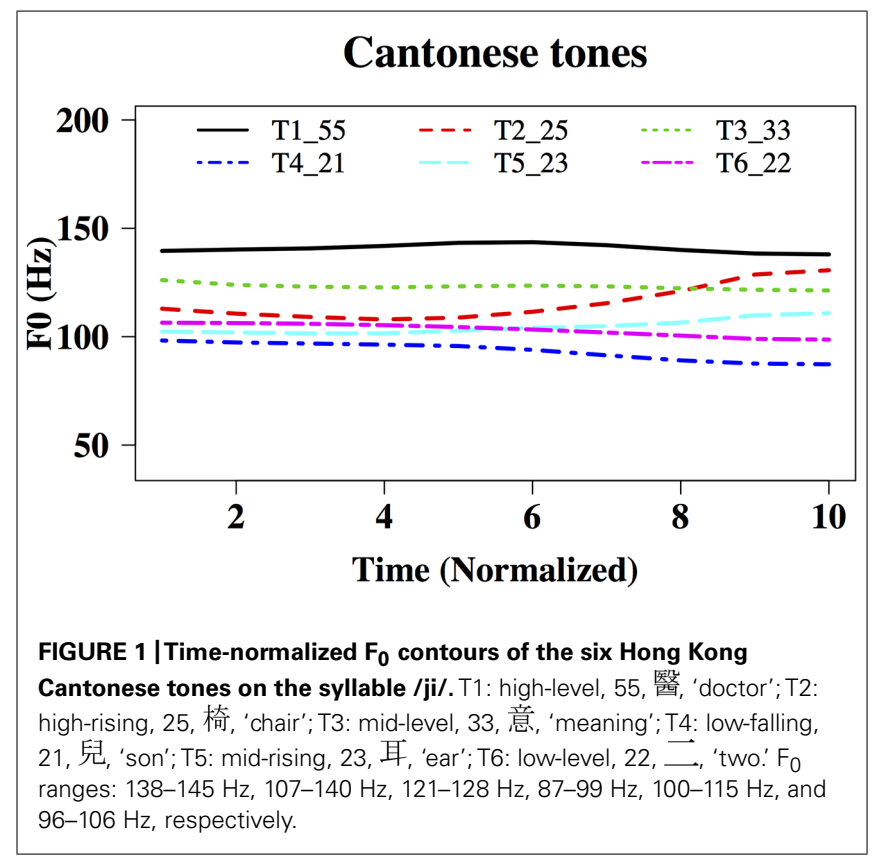

brainstem encoding of speech (in quiet and noise) and musical stimuli (in quiet). During the experiments, participants listened passively to lexical and musical tones while their brain activities were recorded. From the FFR waveforms, we measured the temporal precision, neural tuning/phase-locking, and amplitude of the synchronous neural responses with respect to the periodicity of the stimuli. Apart from FFR recordings, all participants also completed a behavioral task, in which they were required to identify the words that corresponded to the six Cantonese tones. Identification accuracy and response times were calculated.

\section{MATERIALS AND METHODS PARTICIPANTS}

Participants ( $n=14$ in each group) were recruited by advertisements through mass mail services at the Chinese University of Hong Kong. All participants had normal hearing in both ears, with pure-tone air conduction thresholds of $25 \mathrm{~dB}$ HL or better at frequencies of $0.5,1,2$, and $4 \mathrm{kHz}$. All were native speakers of Hong Kong Cantonese, and none reported having speech or hearing disorders or neurological/psychiatric impairments in the questionnaires regarding their music, language, and medical background. Written informed consents were obtained from all participants prior to the experiments. The Institutional Review Board of Northwestern University and The Joint Chinese University of Hong Kong - New Territories East Cluster Clinical Research Ethics Committee approved the study.

All participants were right-handed as assessed by the Edinburgh Handedness Inventory (Oldfield, 1971). The diagnosis of amusia in these participants was conducted using the Montreal Battery of Evaluation of Amusia (MBEA; Peretz et al., 2003), which consists of six subtests measuring the perception of scale, contour, interval, rhythm, and meter of Western melodies, and recognition memory of these melodies. Those who scored 65 or under on the pitch composite score (sum of the scores on the scale, contour, and interval subtests) or below $78 \%$ correct on the MBEA global score were classified as amusic, as these scores correspond to 2 standard deviation below the mean scores of normal controls (Peretz et al., 2003; Liu et al., 2010). As shown in Table S1, 3 out of the 14 amusics (A04, A05, and A13) aged over 40 years, while the rest were between 18 and 22 years. Given that aging has been shown to affect auditory processing abilities (Chisolm et al., 2003), care was taken to find age-matched controls (C07, C08, and $\mathrm{C} 10)$ for the three amusics in their 40s. The other controls (18-24 years) were chosen based on their MBEA global scores (all $>90 \%$ ) and musical training background (matched with the 14 amusics).

Table 1 summarizes the characteristics of the amusic and control groups. As can be seen, amusics performed significantly worse than controls on all MBEA subtests. While the two groups were comparable in sex, handedness, age, and musical training background, controls received more years of education than amusics $[t(26)=2.31, p=0.029]$. This was due to the fact that the three older controls received more years of education than the three older amusics $[t(4)=3.29, p=0.030]$. When the six older participants were excluded, the difference in education background became non-significant between the two groups [amusic 
Table 1 | Characteristics of the amusic ( $n=14,4$ male and 10 female, all right-handed) and control ( $n=14,5$ male and 9 female, all right-handed) groups.

\begin{tabular}{|c|c|c|c|c|c|c|c|c|c|c|c|}
\hline Group & Age & Education & Musical training & Scale & Contour & Interval & Rhythm & Meter & Memory & Pitch composite & MBEA global \\
\hline \multicolumn{12}{|l|}{ Control } \\
\hline Mean & 25.43 & 16.14 & 1.36 & 27.93 & 27.57 & 27.57 & 29.14 & 27.79 & 29.21 & 83.07 & 94.01 \\
\hline SD & 9.51 & 2.68 & 1.95 & 1.69 & 1.87 & 1.70 & 1.61 & 3.12 & 0.80 & 3.08 & 3.25 \\
\hline \multicolumn{12}{|c|}{ Amusic } \\
\hline Mean & 25.50 & 14.07 & 1.07 & 21.93 & 21.57 & 20.00 & 22.93 & 21.14 & 26.71 & 63.50 & 74.60 \\
\hline \multicolumn{12}{|l|}{$t$-test } \\
\hline$t$ & -0.02 & 2.31 & 0.40 & 7.39 & 7.55 & 10.80 & 7.51 & 4.65 & 3.12 & 14.48 & 11.07 \\
\hline$p$ & 0.986 & 0.029 & 0.694 & $<0.001$ & $<0.001$ & $<0.001$ & $<0.001$ & $<0.001$ & 0.004 & $<0.001$ & $<0.001$ \\
\hline
\end{tabular}

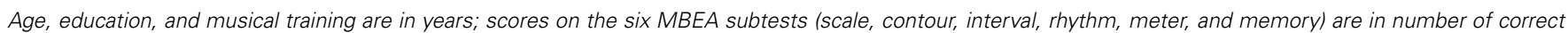

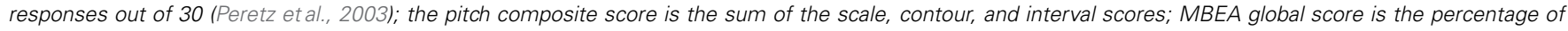
correct responses out of the total 180 trials; $t$ is the statistic of the Welch two sample $t$-test (two-tailed, $d f=26)$.

mean $(\mathrm{SD})=14.36(2.01)$; control mean $(\mathrm{SD})=15.18(1.72)$; $t(20)=1.02, p=0.318]$. In order to account for the possible contribution of education to the current results, years of education were entered as a covariate in the mixed-effects models in Section "Results."

\section{STIMULI}

Speech stimuli consisted of six Cantonese lexical tones spoken on the same syllable /ji/, which led to six different words in Cantonese: Tone 1, 醫, 'doctor'; Tone 2, 椅, 'chair'; Tone 3, 意, 'meaning'; Tone 4, 兒, ‘son'; Tone 5, 耳, 'ear'; Tone 6, 二, 'two.' Using Praat (Boersma and Weenink, 2001), a male native speaker of Hong Kong Cantonese recorded these tones onto a desktop PC in a soundproof booth, using a Shure SM10A headworn microphone and a Roland UA-55 Quad-Capture audio interface at the sampling rate of $44,100 \mathrm{~Hz}$. The six original tones were then duration-normalized to $175 \mathrm{~ms}$ and intensity-normalized to $74 \mathrm{~dB}$ using Praat, so that $\mathrm{F}_{0}$ (fundamental frequency) was the main acoustic feature that differed across the stimuli. Figure 1 shows time-normalized $\mathrm{F}_{0}$ contours of the six tones, ranging from $138-145 \mathrm{~Hz}, 107-140 \mathrm{~Hz}$, $121-128 \mathrm{~Hz}, 87-99 \mathrm{~Hz}, 100-115 \mathrm{~Hz}$, and 96-106 Hz, respectively.

Musical stimuli consisted of two cello tones at different pitches: high $(=150 \mathrm{~Hz})$ versus low $(=112 \mathrm{~Hz})$. These pitches were chosen because they approximated the highest and lowest registers of the lexical tones we used. The two cello tones were derived from a musical sound of a cello being bowed (recorded from a keyboard synthesizer; Musacchia et al., 2007), but with pitches changed to 150 and $112 \mathrm{~Hz}$, duration normalized to $175 \mathrm{~ms}$, and intensity normalized to $75 \mathrm{~dB}$ using Praat.

In the speech in noise condition, Cantonese lexical Tone 1 and Tone 6 (the same as in the speech stimuli) were presented to the participants again, but together with a recursive recording of 10min 6-talker babble noise in Cantonese at signal-to-noise ratio (SNR) levels of 0 and $20 \mathrm{~dB}$ played at the background.

\section{PROCEDURE}

All participants completed three tasks for the present study: (1) behavioral identification and (2) FFR recording of the six
Cantonese tones in quiet, and (3) FFR recording of the cello tones in quiet and Cantonese Tone 1 and Tone 6 in babble noise. The three tasks normally occurred on three different days, separated by several days or months depending on the participants' availability. The order of the three tasks was different across different participants. Participants completed the behavioral tone identification task together with other behavioral tasks (tone discrimination, tone production, and singing) for another study, which in total took about $1 \mathrm{~h}$. The behavioral tone identification task was presented using E-prime 2.0 (Schneider et al., 2002), delivered through Sennheiser HD 380 PRO Headphones and a Roland UA-55 Quad-Capture audio interface at a comfortable listening level. Before the experimental trials, participants were administered a practice session consisting of two repetitions of each of the six Cantonese tones at the duration of $250 \mathrm{~ms}$. In the experimental session, each of the six Cantonese tones used in the FFR task (duration $=175 \mathrm{~ms}$ ) was presented five times in random order. Participants were required to choose the words that corresponded to the tones by clicking one of the six buttons on the computer screen as quickly and accurately as possible. Their responses and reaction times were recorded for later analysis.

Our FFR recording protocol followed closely what has been established in past research (Wong et al., 2007; Song et al., 2008; Skoe and Kraus, 2010), and various efforts had been made to exclude any potential artifacts (see Figure S1 for proof of no artifacts in a "sham" experiment). During FFR recording, participants were encouraged to rest or fall asleep in a recliner chair in an electromagnetically shielded booth with no light on. Stimuli were presented to the participants' right ear through insert earphones (ER-3a, Etymotic Research, Elk Grove Village, IL, USA) at around $80 \mathrm{~dB}$ SPL, using Neuroscan Stim ${ }^{2}$ (Compumedics, El Paso, TX, USA). The order of the six lexical tones was counterbalanced across participants, and that of the cello tones and Tone 1 and Tone 6 in noise was fixed: Tone 1 at $20 \mathrm{~dB}$ SNR, Tone 1 at $0 \mathrm{~dB}$ SNR, Tone 6 at $20 \mathrm{~dB}$ SNR, Tone 6 at $0 \mathrm{~dB}$ SNR, cello tone at $150 \mathrm{~Hz}$, and cello tone at $112 \mathrm{~Hz}$. The interstimulus-interval jittered between 74 and 104 ms. Responses were collected using CURRY Scan 7 Neuroimaging Suite (Neuroscan, 
Compumedics, El Paso, TX, USA) with four $\mathrm{Ag}-\mathrm{AgCl}$ scalp electrodes, differentially recorded from vertex $(\mathrm{Cz}$, active) to bilateral linked mastoids (M1+M2, references), with the forehead (Fpz) as ground. Contact impedance was less than $5 \mathrm{k} \Omega$ for all electrodes. For each stimulus, two blocks of 1500 sweeps were collected at each polarity with a sampling rate of $20,000 \mathrm{~Hz}$, lasting around $12 \mathrm{~min}$.

Filtering, artifact rejection, and averaging were performed offline using CURRY 7. Responses were band-pass filtered from 80 to $5000 \mathrm{~Hz}, 12 \mathrm{~dB} /$ octave, and trials with activity greater than $\pm 35 \mu \mathrm{V}$ were considered artifacts and rejected. Waveforms were averaged with a recording time window spanning $50 \mathrm{~ms}$ prior to the onset and $50 \mathrm{~ms}$ after the offset of the stimulus. Responses of alternating polarity were then added together to isolate the neural response by minimizing stimulus artifact and cochlear microphonic (Russo et al., 2004; Aiken and Picton, 2008).

\section{DATA ANALYSIS}

Percentage of correct responses and reaction time were calculated for the behavioral tone identification task. Only trials with correct responses were retained in the reaction time analysis.

Main FFR data were analyzed using Matlab (The Mathworks, Natick, MA, USA) scripts adapted from the Brainstem Toolbox (Skoe et al., 2013). Before analysis, the stimuli were resampled to $20,000 \mathrm{~Hz}$, to match the sampling rate of the responses. Both the stimuli and responses were band-pass filtered from 80 to $2500 \mathrm{~Hz}$ to remove the slower cortical ERPs and attenuate EEG noise above the limit of phase-locking (Skoe and Kraus, 2010; Bidelman et al., 2013). The FFR was assumed to encompass the entire duration of the stimulus (175 ms).

Using a sliding window analysis procedure (Wong et al., 2007; Song et al., 2008), 50-ms bins of the FFR were shifted in 1-ms steps to produce a total of $125(=175-50)$ Hanning-windowed overlapping bins in the frequency domain. A narrow-band spectrogram was calculated for each FFR bin by applying the fast Fourier transform (FFT). To increase spectral resolution, each time bin was zero-padded to $1 \mathrm{~s}$ before performing the FFT. The spectrogram gave an estimate of spectral energy over time and the $\mathrm{F}_{0}$ (pitch) contour was extracted from the spectrogram by finding the spectral peak closest to the expected (stimulus) frequency. Both $\mathrm{F}_{0}$ frequency and amplitude were recorded for each time bin. The same short-term spectral analysis procedure was applied to the stimulus waveforms, in order to compare the responses with the stimuli. The following time, pitch, and amplitude measurements were extracted to determine how well the amusic brainstem encodes the timing, periodicity, and the spectral envelop of the evoking stimuli compared to controls (Wong etal., 2007; Song etal., 2008; Skoe and Kraus, 2010; Skoe et al., 2013).

Neural lag (in ms) is the amount of time shift to achieve the maximum positive correlation between the waveforms of the stimulus and the response. This measure was calculated using a cross-correlation technique that slid the stimulus and response waveforms back and forth in time with respect to one another until the maximum positive correlation between the two was found. It estimated the FFR latency due to the neural conduction time of the auditory system for each tone/participant, which was taken into account when calculating each of the following measurements.

Pitch strength (or autocorrelation, values between -1 and 1 ) is a measure of periodicity and phase locking of the response. Using a short-time running autocorrelation technique, the response, in 50-ms time bins, was successively time-shifted in 1-ms steps with a delayed version of itself, and a Pearson's $r$ was calculated at each 1-ms interval. The maximum (peak) autocorrelation value was recorded for each bin, with higher values indicating more periodic time frames. Pitch strength was calculated by averaging the autocorrelation peaks ( $r$-values) from the 125 bins for each tone/participant.

Pitch error (in $\mathrm{Hz}$ ) is the average absolute Euclidian distance between the stimulus $\mathrm{F}_{0}$ and response $\mathrm{F}_{0}$ across the total of 125 time bins analyzed. It is a measure of pitch encoding accuracy of the FFR over the entire duration of the stimulus, shifted in time to match with the response based on the specific neural lag value obtained for each tone/participant.

Stimulus-to-response correlation (values between -1 and 1 ) is the Pearson's correlation coefficient $(r)$ between the stimulus and response $\mathrm{F}_{0}$ contours (shifted in time by neural lag). This measure indicates both the strength and direction of the linear relationship between the two signals.

Root mean square (RMS) amplitude (in $\mu \mathrm{V}$ ) of the FFR waveform is the magnitude of neural activation over the entire FFR period (neural lag, neural lag $+175 \mathrm{~ms}$ ).

Signal-to-noise ratio is the ratio of the RMS amplitude of the response over the RMS of the pre-stimulus period $(50 \mathrm{~ms})$.

Mean amplitudes of the first three harmonics (in $\mathrm{dB}$, indicating peak amplitudes of the power spectrum) are spectral peaks within the frequency ranges of the fundamental frequency $\left(\mathrm{F}_{0}\right.$, first harmonic) and the next two harmonics (second and third harmonics). They were calculated by finding the largest spectral peaks in the frequency ranges of the first three harmonics in the narrow-band spectrogram after applying the short-time Fourier transform (STFT).

Statistical analyses were conducted using R (R Core Team, 2014). For parametric statistical analyses, $r$-values (pitch strength, stimulus-to-response correlation) were converted to $z^{\prime}$-scores using Fisher's transformation (Wong et al., 2007), percent correct scores for tone identification was converted using rationalized arcsine transformation (Studebaker, 1985), and reaction times were transformed using log transformation (Howell, 2009), since these measures deviated from normal distributions (Shapiro-Wilk normality test: all $p s<0.05)$. Linear mixed-effects models were fit on all measures using the R package 'nlme' (Pinheiro and Bates, 2009). Post hoc pairwise comparisons were conducted using $t$-tests with $p$-values adjusted with the Holm (1979) method.

\section{RESULTS \\ BRAINSTEM ENCODING OF SPEECH (IN QUIET AND NOISE) AND MUSICAL STIMULI (IN OUIET)}

Figure 2 shows the waveforms of the six Cantonese lexical tones, and grand average waveforms of the FFRs to the six tones (heard in quiet) of the amusic and control groups. Figure 3 shows pitch tracks of the original stimuli (black lines), and those of the responses (yellow lines) from both groups. In the pitch track plots, 
A1
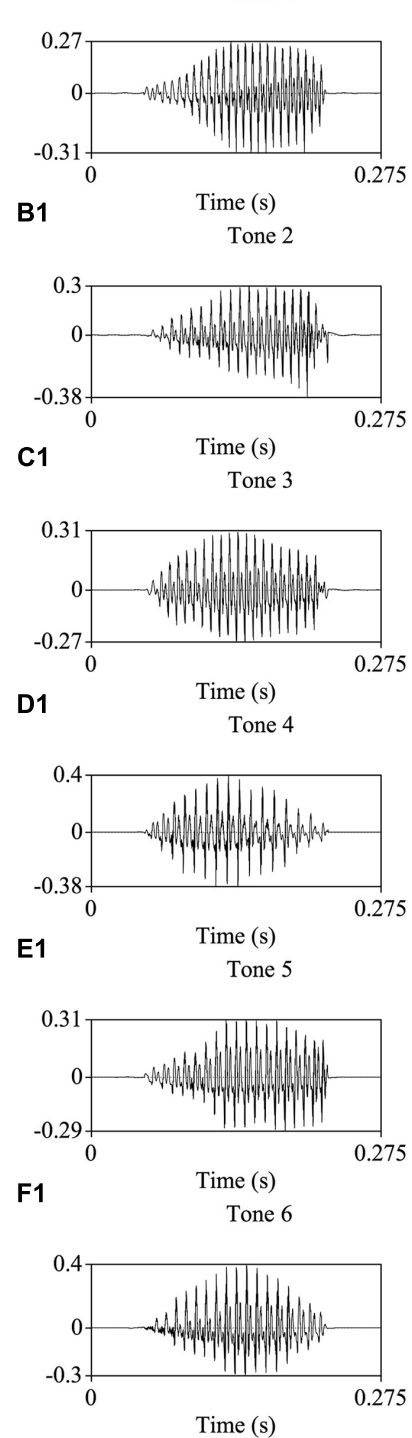

A2
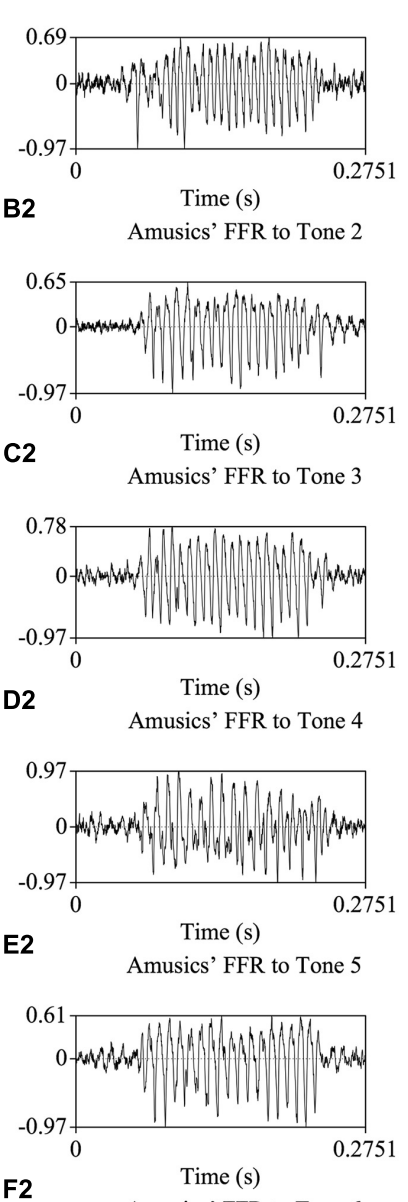

F2

$$
\text { Amusics' FFR to Tone } 6
$$

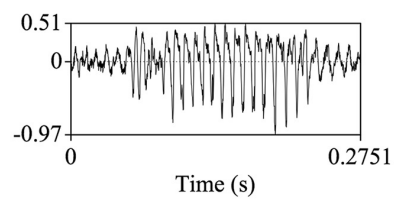

A3

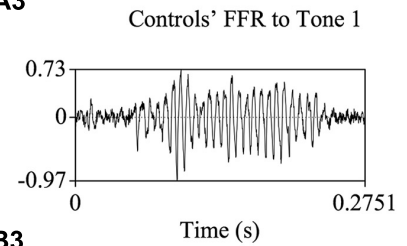

B3

Controls' FFR to Tone 2
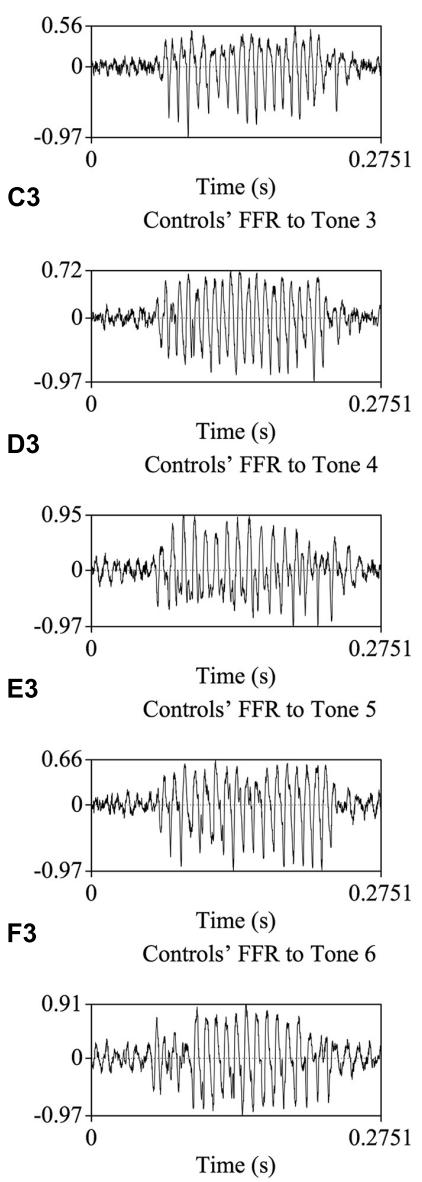

FIGURE 2 | Waveforms of the original stimuli /ji/ with six Cantonese tones (A1-F1), and those of the grand-average FFRs of amusics (A2-F2) and controls (A3-F3).

the small red dots signify regions where the extracted $\mathrm{F}_{0} \mathrm{~s}$ were below the noise floor (i.e., SNR was less than one, reflecting the magnitude of the response $\mathrm{F}_{0}$ ), and the small blue dots indicate regions where the extracted $\mathrm{F}_{0} \mathrm{~s}$ were not at the spectral maximum (indicating weak $\mathrm{F}_{0}$ encoding strengths; Song et al., 2008; Skoe et al., 2013).

Figure 4 shows grand average waveforms of amusics' and controls' FFRs to Cantonese Tone 1 and Tone 6 heard in babble noise ( $\mathrm{SNR}=0$ and $20 \mathrm{~dB}$ ), and the corresponding pitch tracks. Figure 5 shows the waveforms of the two cello tones, and grand average waveforms of the FFRs to the cello tones (heard in quiet) of both groups, and the corresponding pitch tracks.

Linear mixed-effects models were first fit on all FFR measures for each experimental condition (speech-in-quiet, speech-in-noise, music), with group (amusic, control), tone (Tones $1-6$ in the speech-in-quiet condition, Tone 1 versus Tone 6 in the speech-in-noise condition, and high versus low cello tone in the music condition), and noise (SNR $=0$ and $20 \mathrm{~dB}$ in the speech-in-noise condition) as fixed effects, education (in years) as a covariate, and participants (and tones nested in participants) as random effects. Detailed results are shown in Tables S2-S4.

Among all measures/conditions, a significant group effect $[F(1,25)=5.11, p=0.033]$ was only observed for the amplitude of the first harmonic $\left(\mathrm{F}_{0}\right)$ in the speech-in-noise condition, with controls showing larger $\mathrm{F}_{0}$ amplitudes in FFRs to tones in noise than amusics (Table S3). Although the effects of tone and noise were significant in many cases, no tone $\times$ noise or tone $x$ noise $\times$ group interaction was observed for any measures 


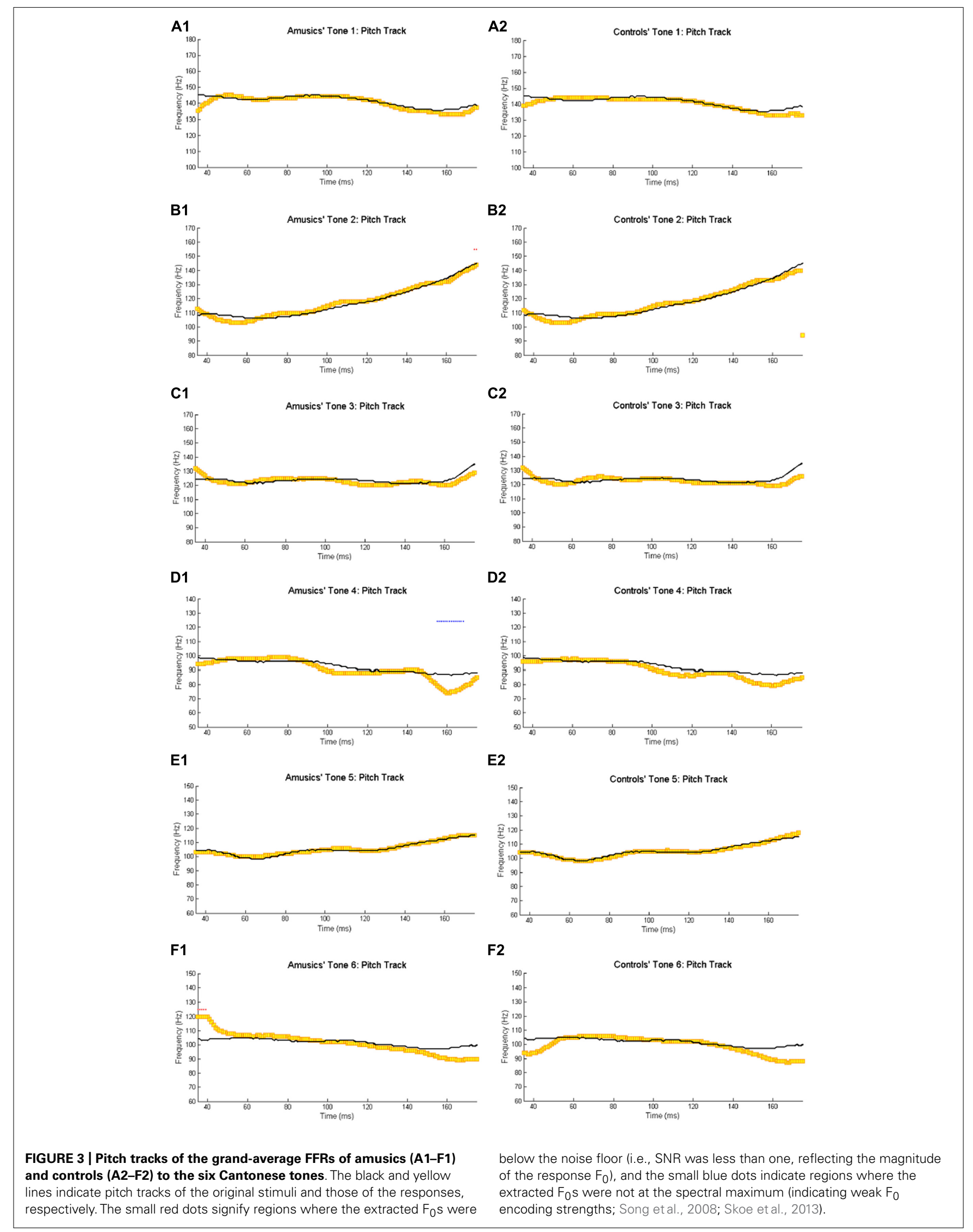




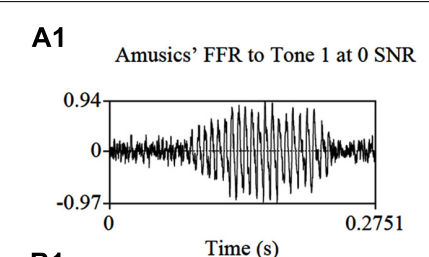

B1 Amusics' FFR to Tone 1 at 20 SNR

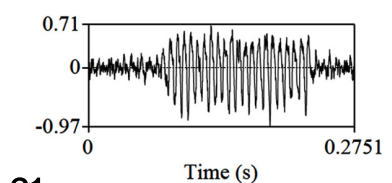

C1 Amusics' FFR to Tone 6 at 0 SNR

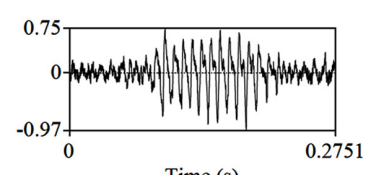

D1 Time (s)

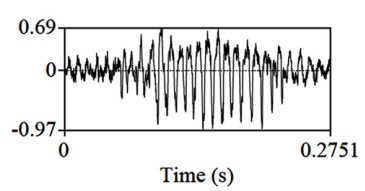

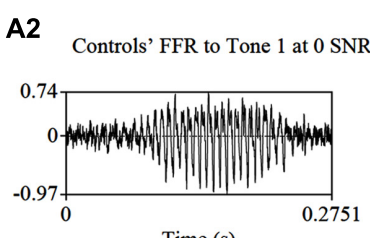

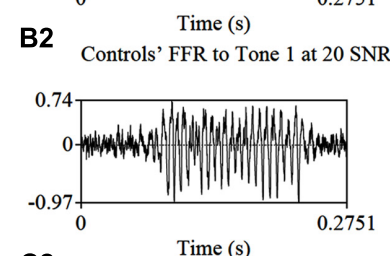

C2 Controls' FFR to Tone 6 at 0 SNR

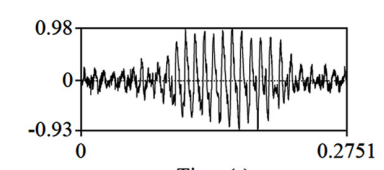

D2 Controls' FFR to Tone 6 at 20 SNR

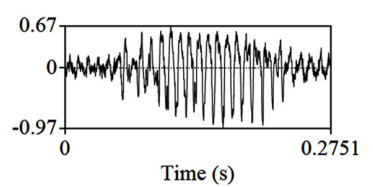

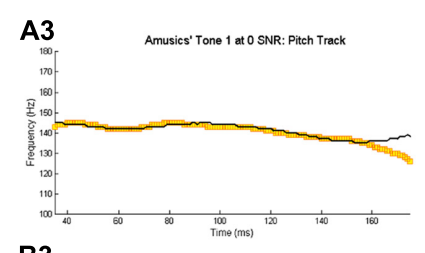
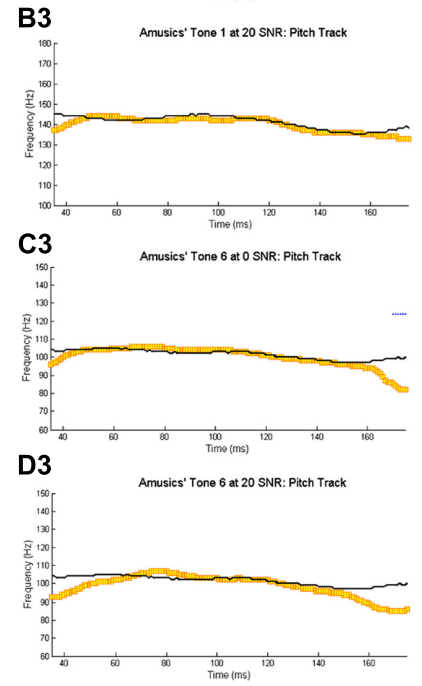

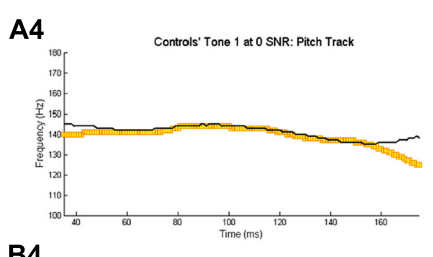

B4
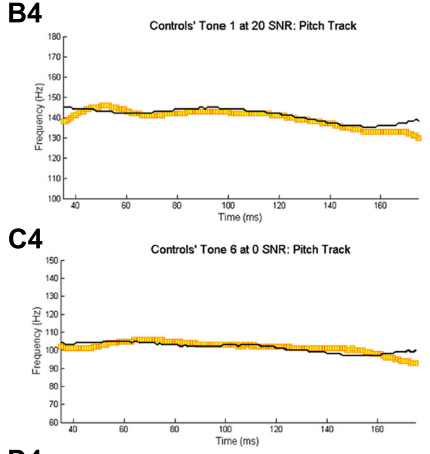

D4

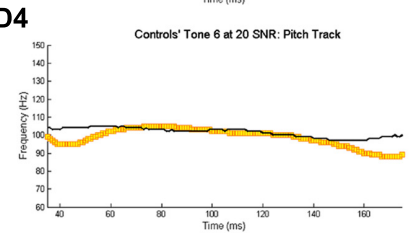

FIGURE 4 | Grand average waveforms of amusics' (A1-D1) and controls' (A2-D2) FFRs to Cantonese Tone 1 and Tone 6 heard in babble noise (SNR = 0 and $20 \mathrm{~dB}$ ), and the corresponding pitch tracks (A3-D3, A4-D4).

in the speech-in-noise condition (Table S3), or tone $\times$ group interaction in the speech-in-quiet (Table S2) or music condition (Table S4). The only significant tone $\times$ group interaction $[F(1,26)=4.65, p=0.040]$ was observed for neural lag in the speech-in-noise condition: controls showed longer neural lags than amusics for Tone 1 in noise, $t(54)=-3.09, p=0.003$, but not for Tone 6 in noise, $t(54)=0.90, p=0.370$ (Table S3). The only significant group $\times$ noise interaction $[F(1,52)=7.19$, $p=0.010]$ was observed for pitch error in the speech-in-noise condition, as controls showed smaller pitch errors than amusics when $\mathrm{SNR}=0 \mathrm{~dB}, t(54)=1.67, p=0.101$, but larger pitch errors when $\mathrm{SNR}=20 \mathrm{~dB}, t(54)=-0.53, p=0.597$ (Table S3).

Together, these results suggest that amusics and controls showed largely comparable FFRs under different tone and noise conditions. The significant group difference in $\mathrm{F}_{0}$ amplitude $(p=0.033)$ and tone $\times$ group interaction on neural lag $(p=0.040)$ and group $\times$ noise interaction on pitch error $(p=0.010)$ in the speech-in-noise condition were likely due to familywise Type I errors (false positives) from multiple comparisons, as these effects would become non-significant if using Bonferroni correction to adjust for the significance level $[p=0.05 /(9$ measures $\times 3$ conditions $)=0.0019]$. Furthermore, the uneven experimental design of the tone (six levels in speech-in-quiet, two levels in speech-in-noise, and two levels in the music condition) and noise factors (two levels in speech-in-noise) gave rise to different numbers of multiple observations (replications) for each participant in each experimental condition, which would make unequal contributions to the comparison of treatments applied to different conditions (Mead, 1990). Thus, for statistical analysis and in the interest of space, FFR measures from different tones in the three experimental conditions and different noise levels in the speech-in-noise condition were pooled and averaged for each participant in the following analyses, in order to examine the overall effects of group (amusic, control) and condition (speech-in-quiet, speech-in-noise, music) on each FFR measure.

Table 2 lists the results from the linear mixed-effects models on each FFR measure (averaged across different tones in each condition, and across the two noise levels in the speech-in-noise condition), with group (amusic, control) and condition (speechin-quiet, speech-in-noise, music) as fixed effects, education (in years) as a covariate, and participants (and conditions nested in participants) as random effects. No significant effect of group or condition $\times$ group interaction was observed for any measures. The effect of condition (speech-in-quiet, speech-in-noise, music) was significant for all measures, and participants' years of education negatively affected pitch strengths and SNRs of their FFRs. These significant effects are discussed in detail below (Figures 6-9). In the interest of space, non-significant effects are omitted from discussion.

Figure 6A shows mean neural lags (in ms) of FFRs under the three experimental conditions (speech-in-quiet, speech-in-noise, music) for amusics and controls. There was a significant effect of condition (Table 2), as both groups exhibited longer neural lags for musical than speech-in-noise $(p<0.001)$ and speech in-quiet stimuli $(p<0.001)$. 
A1
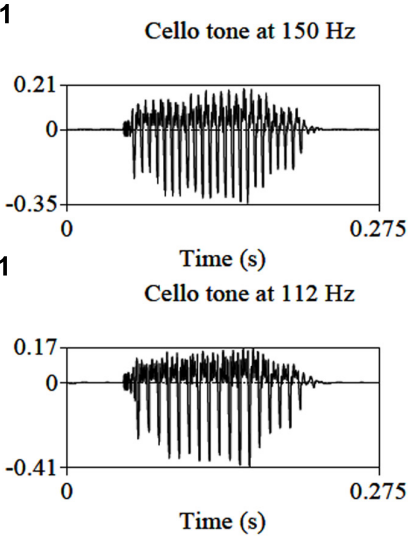

C1

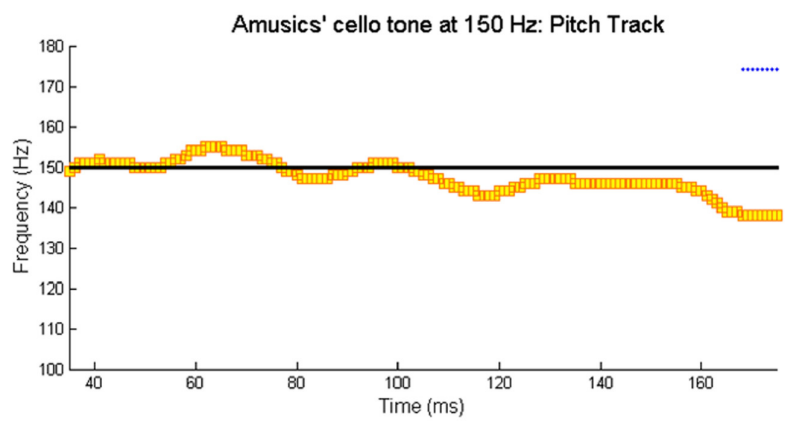

D1

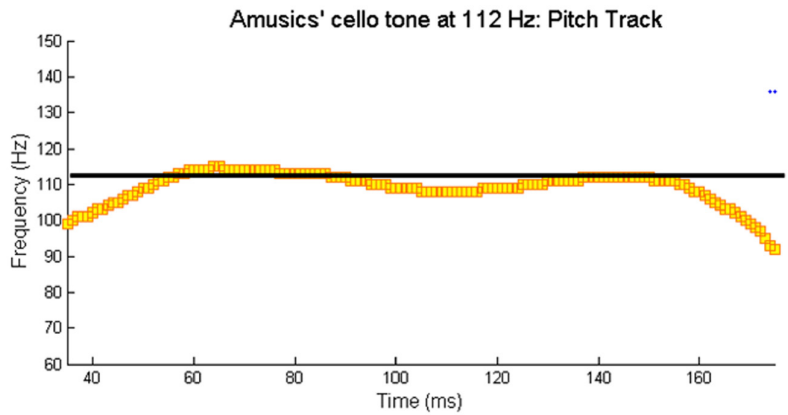

A2

Amusics' FFR to cello tone at $150 \mathrm{~Hz}$

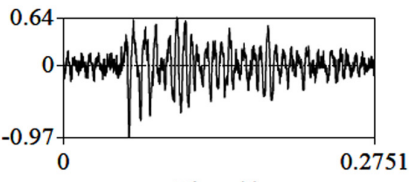

Time (s)

Amusics' FFR to cello tone at $112 \mathrm{~Hz}$

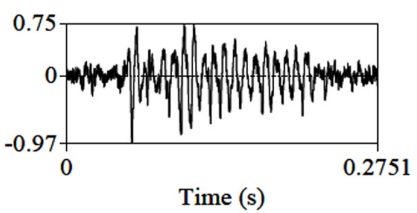

A3

Controls' FFR to cello tone at $150 \mathrm{~Hz}$

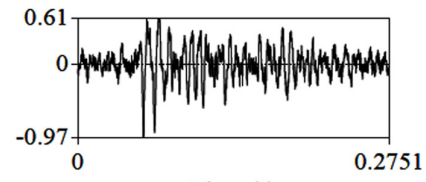

B3

Time (s)

Controls' FFR to cello tone at $112 \mathrm{~Hz}$

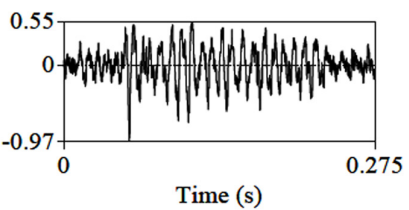

C2

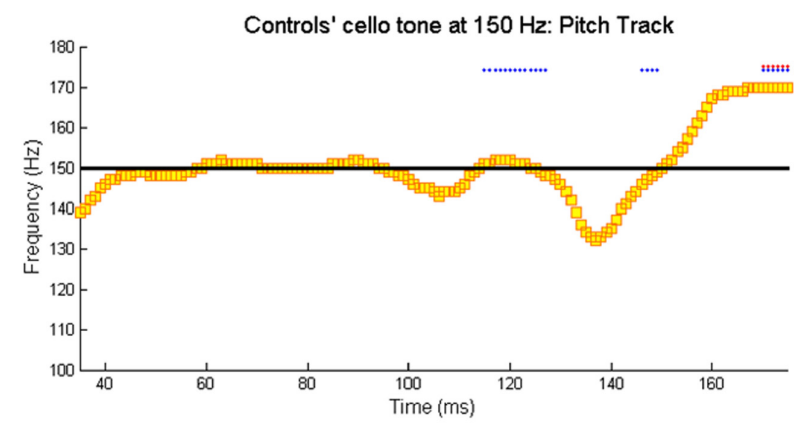

D2

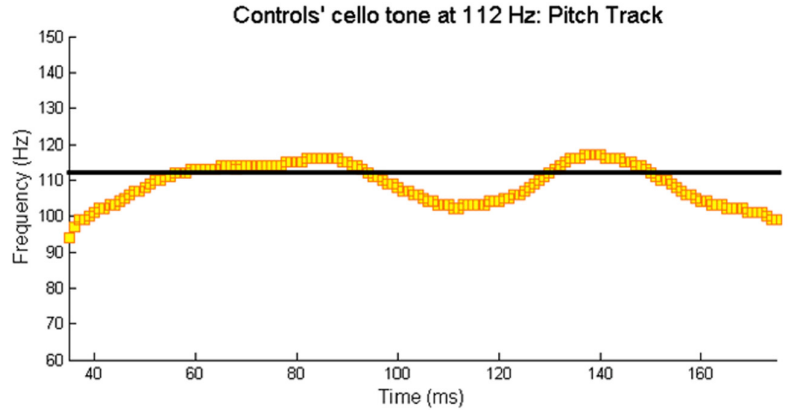

FIGURE 5 | Waveforms of the original two cello tones (A1,B1), and grand average waveforms of the FFRs to the cello tones (heard in quiet) of the amusic $(A 2, B 2)$ and control $(A 3, B 3)$ groups, and the corresponding pitch tracks $(C 1, C 2, D 1, D 2)$.

Figure 6B shows mean pitch strengths (autocorrelations) of FFRs under the three experimental conditions for the two groups. The linear mixed-effects model on Fisher-transformed $z^{\prime}$-scores revealed a main effect of condition (Table 2 ), as pitch strengths of both groups were the highest in the speech-in-quiet condition and the lowest in the music condition, with those of the speech-in-noise condition in between (all ps $<0.001$ ). The effect of education on pitch strength was also significant (Table 2): the more education participants received, the lower their pitch strengths. This was confirmed by the negative correlation between participants' education background (in years) and their pitch strengths across all conditions $[r(82)=-0.19$, $p=0.079]$.
Given that neural pitch strength of the FFR varies dramatically between steady-state and dynamic segments of the tones (Krishnan et al., 2010b; Bidelman et al., 2011a), we divided each of the tones into seven 25-ms sections and calculated mean pitch strengths of amusics and controls for each section using Krishnan et al. (2010b) and Bidelman et al. (2011a) methods. As can be seen from Table 3, among the total 84 ( 7 sections $\times 12$ tones) pairwise comparisons, only 2 (section 1 of Tone 1 in quiet, section 3 of Tone 1 at 0 SNR) demonstrated significant group differences in pitch strength. These differences would become non-significant if using Bonferroni correction to adjust for the significance level ( $p=0.05 / 84=0.0006)$ in order to prevent familywise Type I errors (false positives). In general, these results suggest that amusics did 
Table 2 | Results from the mixed-effects models on the effects of Condition [speech-in-quiet, speech-in-noise, music, $F(2,52)], G r o u p$ [amusic versus control, $F(1,25)]$, Education $[F(1,25)]$, and Condition $\times$ Group interaction $[F(2,52)]$ on measures of FFRs.

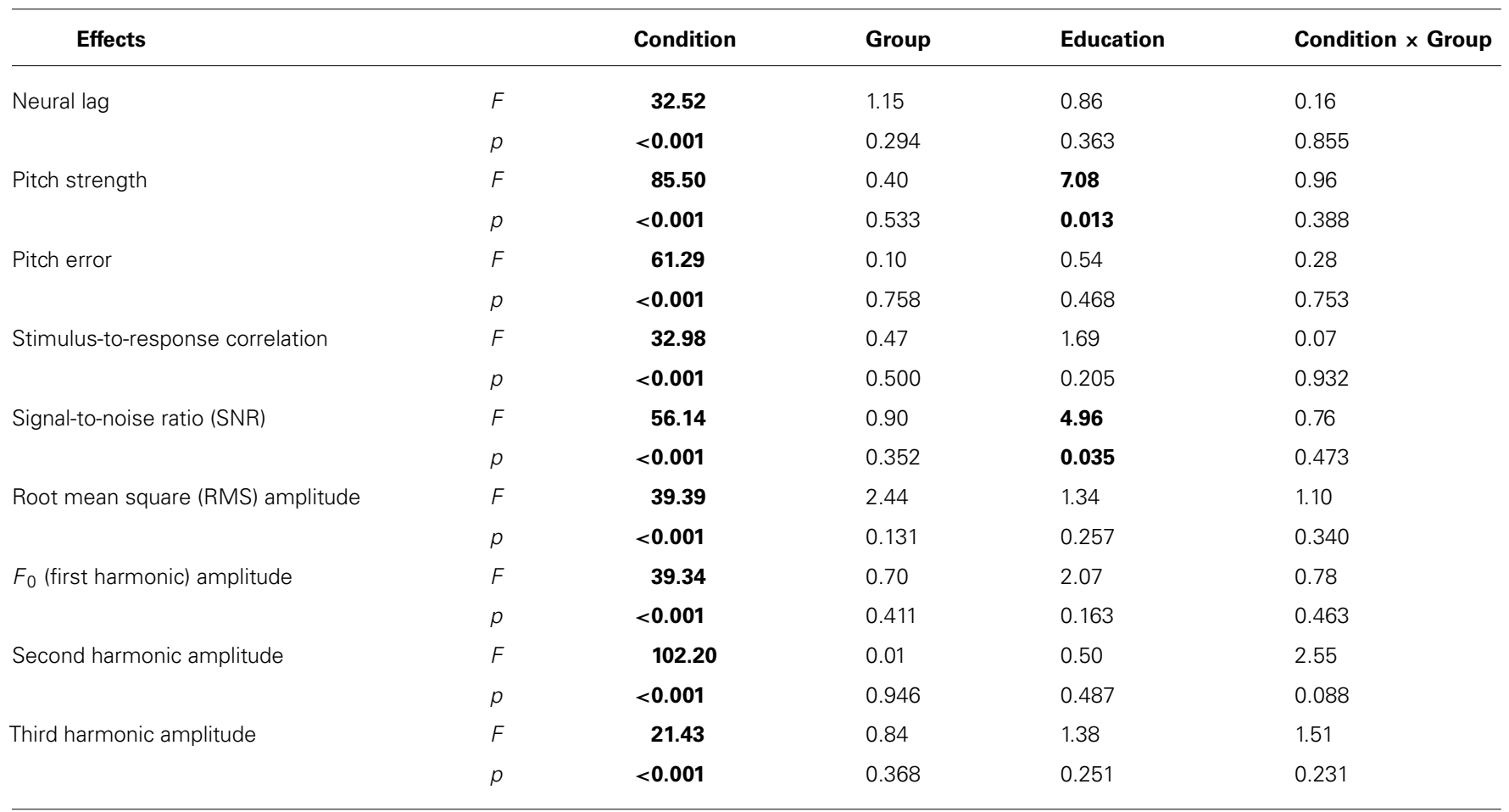

Significant effects are highlighted in boldface.
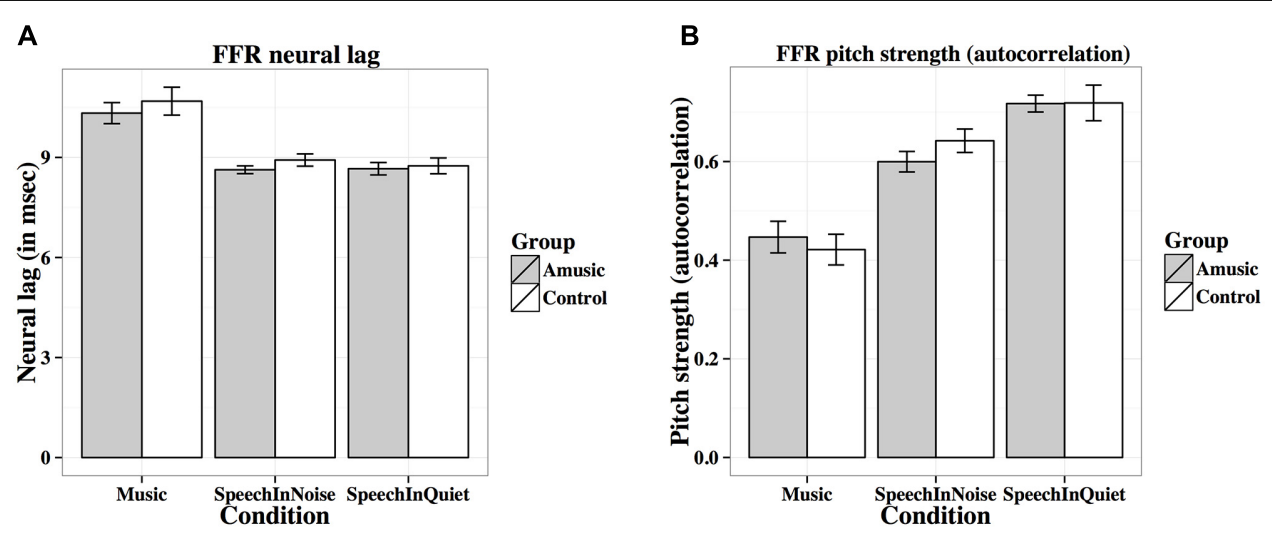

FIGURE 6 | Frequency-following response (FFR) neural lags ( $A$; in $\mathrm{ms}$ ) and pitch strengths (B; autocorrelations) of amusics and controls under the three experimental conditions (music, speech-in-noise, speech-in-quiet).

not differ from controls in FFR pitch strength across different sections of the tones in speech-in-quiet, speech-in-noise, or music conditions.

Figure 7A shows mean pitch errors (in $\mathrm{Hz}$ ) of FFRs under the three experimental conditions for amusics and controls. There was a significant effect of condition (Table 2), as both groups showed larger pitch errors for musical than speech-in-noise and speech-in-quiet stimuli (both $p s<0.001$ ).

Figure 7B shows mean stimulus-to-response correlations of the three types of stimuli for the two groups. The linear mixed-effects model on Fisher-transformed $z^{\prime}$-scores revealed a main effect of condition (Table 2), as both groups demonstrated lower stimulusto-response correlations for musical than speech-in-noise and speech-in-quiet stimuli (both $p s<0.001$ ), and lower stimulusto-response correlations for speech-in-noise than speech-in-quiet stimuli $(p=0.048)$.

Figure 8A shows mean SNRs of FFRs under the three experimental conditions for amusics and controls. SNR differed significantly across different experimental conditions (Table 2), in the order of music $<$ speech-in-noise $<$ speech-in-quiet for 
Table 3 | Mean pitch strengths of amusics and controls over seven 25-ms sections of the six Cantonese tones heard in quiet, Cantonese Tone 1 and Tone 6 heard in noise (SNR $=0$ and $20 \mathrm{~dB}$ ), and cello tones at 150 and $112 \mathrm{~Hz}$.

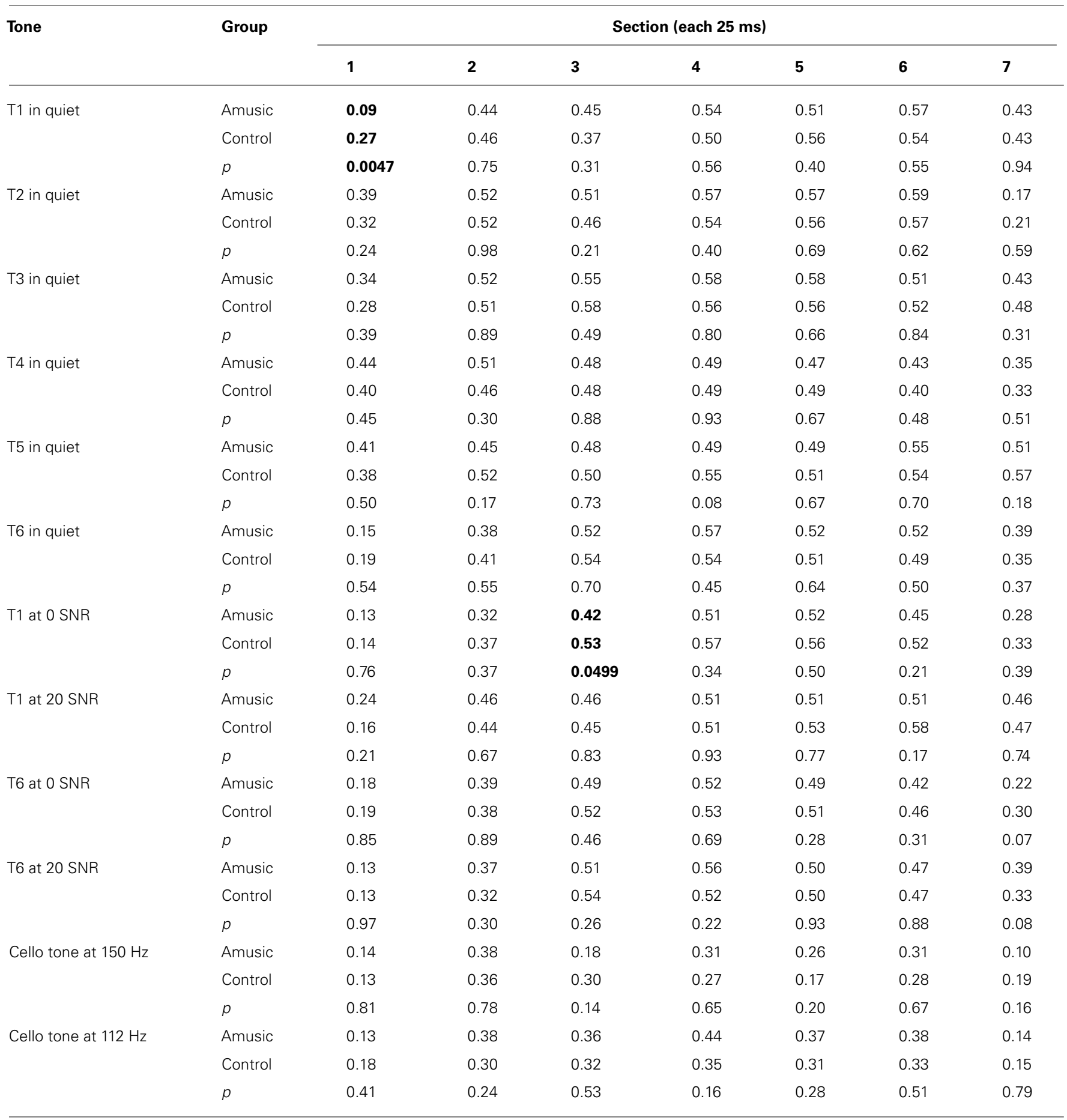

The p-values were obtained from Welch two sample t-tests (two-tailed, $d f=26$ ). Sections with significant group differences are highlighted in boldface. Note that the pitch strength values in this table are different from those in Figure 6B due to different calculation methods used (Krishnan et al., 2010b; Bidelman et al., 2011a; Skoe etal., 2013).

both groups (all ps $<0.05)$. There was also a significant effect of education on SNR (Table 2): the more education participants received, the lower their SNRs. This was confirmed by the negative correlation between participants' education background (in years) and their SNRs across all conditions $[r(82)=-0.20$, $p=0.075]$.

Figure 8B shows mean RMS amplitudes (in $\mu \mathrm{V}$ ) of the FFR waveforms under the three experimental conditions for 

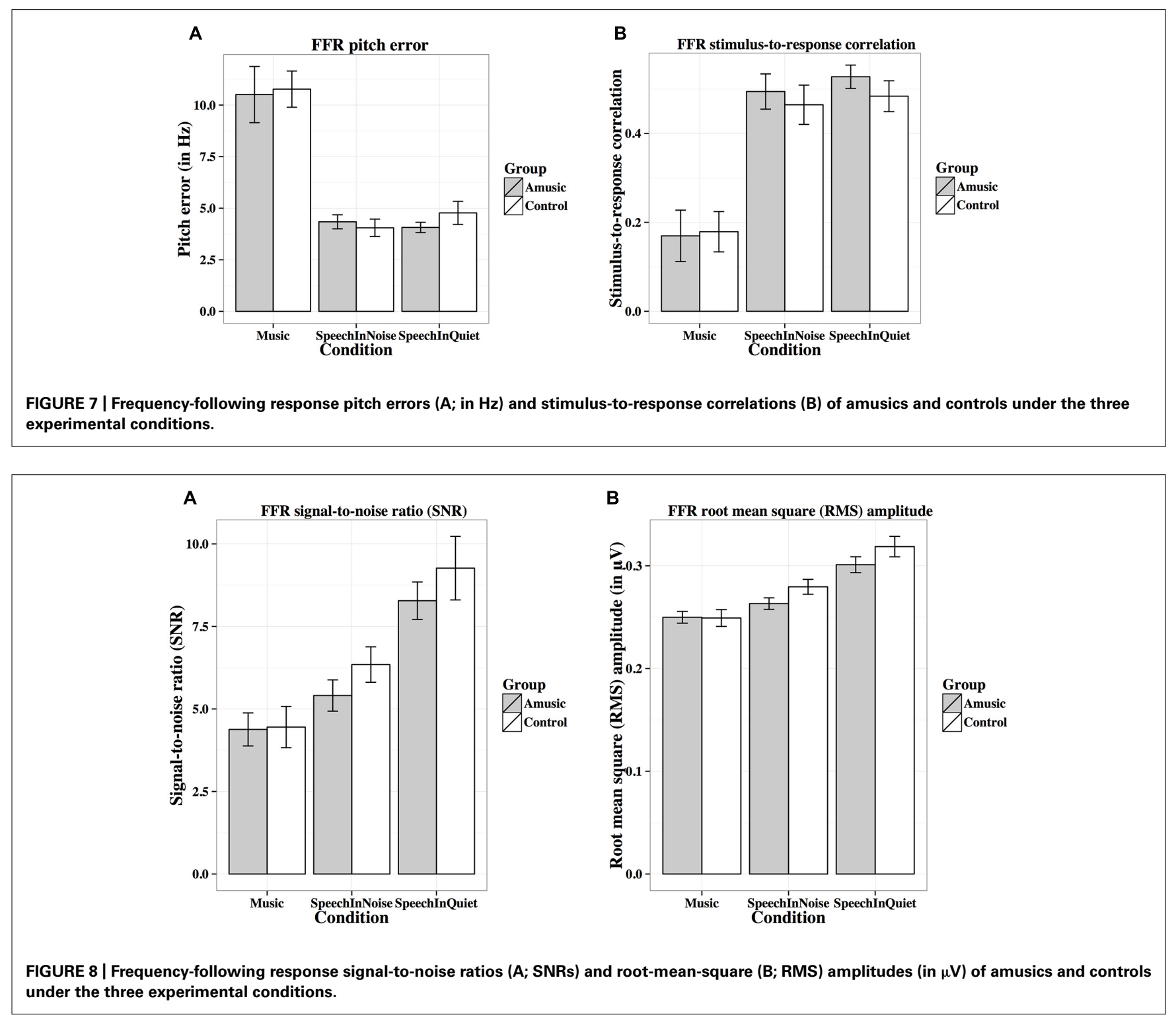

amusics and controls. RMS amplitudes differed significantly across different experimental conditions (Table 2), in the order of music $<$ speech-in-noise $<$ speech-in-quiet for both groups (all ps $<0.01$ ).

Figure 9 shows mean amplitudes of spectral peaks within the frequency ranges of the fundamental frequency $\left(\mathrm{F}_{0}\right.$, first harmonic) and the next two harmonics (second and third harmonics) under the three experimental conditions for amusics and controls. Mean amplitudes of these harmonics were significantly affected by different experimental conditions (Table 2), as both groups showed lower harmonic amplitudes for music than speech-in-noise/speech-in-quiet stimuli (all ps $<0.01$ ).

BEHAVIORAL IDENTIFICATION OF THE SIX CANTONESE LEXICAL TONES Figure 10A shows percent-correct scores of amusics and controls for the behavioral tone identification task. A linear mixedeffects model was fit on rationalized arcsine transformed scores, with group (amusic, control) and tone (Tones 1-6) as fixed effects, education (in years) as a covariate, and participants (and tones nested in participants) as random effects. Tone identification scores differed significantly across different tones $[F(5,130)=4.64, p=0.002$ with the Greenhouse-Geisser correction], with Tone 5 receiving significantly worse identification than Tone $1(p=0.025)$. There was a significant main effect of group $[F(1,25)=4.81, p=0.038]$, as controls achieved better tone identification performance than amusics. A significant effect of education was also observed for tone identification $[F(1,25)=4.73, p=0.039]$ : the more education participants received, the worse their tone identification. This was confirmed by the negative correlation between participants' education background (in years) and their mean tone identification scores across the six tones $[r(26)=-0.18, p=0.351]$. No significant tone $\times$ group interaction was observed $[F(5,130)=1.15, p=0.337$ with the Greenhouse-Geisser correction]. 

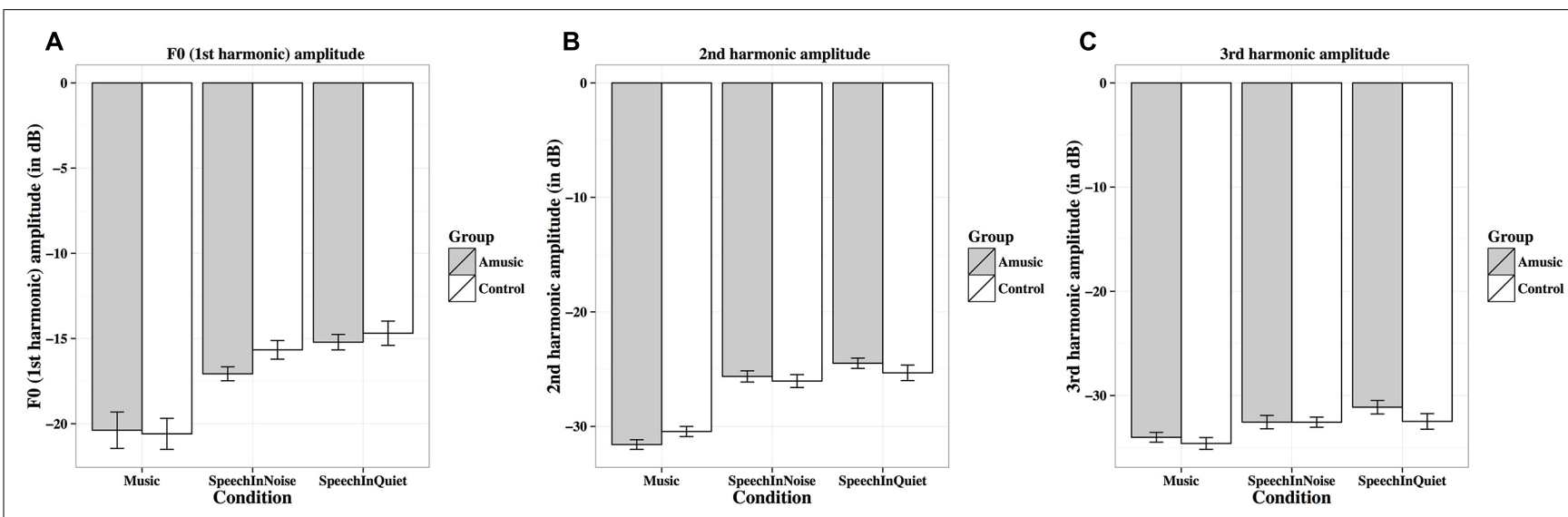

FIGURE 9 | Frequency-following response first (A), second (B), and third (C) harmonic amplitudes (in dB) of amusics and controls under the three experimental conditions.
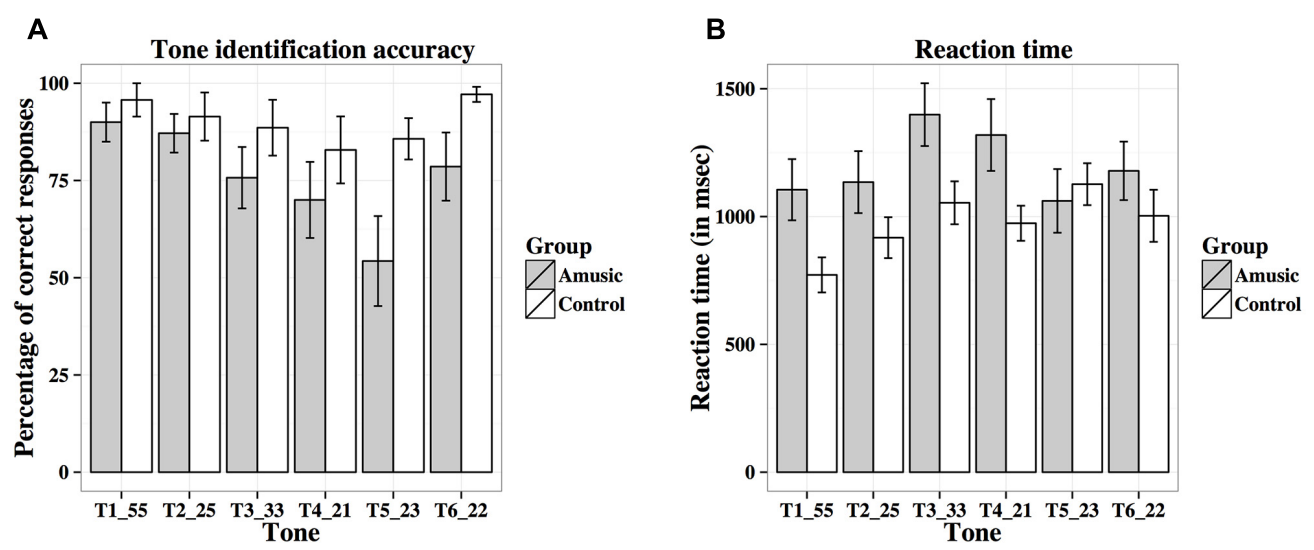

FIGURE 10 | Percent-correct scores (A) and reaction times (B; in ms) of amusics and controls for the behavioral tone identification task.

Figure 10B shows reaction times of amusics and controls on the correct trials for the identification of the six tones. Among the 28 participants, five participants (four amusics and one control) scored $0 \%$ correct on some tones (Tones 3-5), thus leading to missing data. The linear mixed-effects model on logtransformed reaction times indicated that reaction times differed significantly across different tones $[F(5,123)=3.77, p=0.003$; or $F(5,105)=3.01, p=0.016$ with the Greenhouse-Geisser correction and when participants with missing data were removed], as Tone 1 elicited significantly shorter reaction times than Tones $3-5$ (all ps $<0.05$ ). The other effects were non-significant [Group: $F(1,25)=1.52, p=0.229$; Education: $F(1,25)=0.03, p=0.856$; Tone $\times$ Group: $F(5,123)=1.00, p=0.423$; or $F(5,105)=0.83$, $p=0.522$ with the Greenhouse-Geisser correction and when participants with missing data were removed].

Table 4 shows confusion matrices of tone identification of the two groups. Fisher's Exact Test for Count Data (two-tailed) revealed significant differences in confusion patterns between amusics and controls for Tone $4(p=0.006)$, Tone $5(p<0.001)$, and Tone $6(p=0.012)$. Amusics were more likely than controls to confuse between tones that shared similar acoustic features, e.g., Tones 2 and 5 (two rising tones), Tones 3 and 6 (two level tones), and Tones 4 and 6 (two low tones).

\section{CORRELATION ANALYSES}

Correlation analyses were conducted between brainstem encoding and behavioral identification of the six Cantonese lexical tones heard in quiet in order to examine whether there was any association between neural and cognitive processing of lexical tones in the current participants. Results revealed no significant correlation between FFR pitch measures (pitch strength, pitch error, stimulus-to-response correlation) and tone identification accuracy, or between FFR neural lag and tone identification response time when all participants were analyzed together or for each group alone (all ps > 0.05).

Correlation analyses were conducted between MBEA global scores and FFR/behavioral measures of lexical tone processing in quiet in order to examine whether there was any association between music perception and lexical tone processing in the current participants. A significant positive correlation was 
Table 4 | Confusion matrices of tone identification of amusics and controls.

\begin{tabular}{lllllll}
\hline & T1_55 & T2_25 & T3_33 & T4_21 & T5_23 & T6_22 \\
\hline Amusic & & & & & & \\
T1_55 & $\mathbf{9 0 . 0 0}$ & 0.00 & 7.14 & 0.00 & 0.00 & 0.00 \\
T2_25 & 1.43 & $\mathbf{8 7 . 1 4}$ & 4.29 & 2.86 & 40.00 & 4.29 \\
T3_33 & 4.29 & 0.00 & $\mathbf{7 5 . 7 1}$ & 7.14 & 2.86 & 7.14 \\
T4_21 & 0.00 & 1.43 & 0.00 & $\mathbf{7 0 . 0 0}$ & 1.43 & 7.14 \\
T5_23 & 1.43 & 10.00 & 4.29 & 2.86 & $\mathbf{5 4 . 2 9}$ & 0.00 \\
T6_22 & 0.00 & 1.43 & 7.14 & 17.14 & 1.43 & $\mathbf{7 8 . 5 7}$ \\
No response & 2.86 & 0.00 & 1.43 & 0.00 & 0.00 & 2.86 \\
Control & & & & & & \\
T1_55 & $\mathbf{9 5 . 7 1}$ & 0.00 & 7.14 & 7.14 & 0.00 & 0.00 \\
T2_25 & 0.00 & $\mathbf{9 1 . 4 3}$ & 1.43 & 0.00 & 12.86 & 0.00 \\
T3_33 & 4.29 & 0.00 & $\mathbf{8 8 . 5 7}$ & 1.43 & 0.00 & 1.43 \\
T4_21 & 0.00 & 0.00 & 0.00 & $\mathbf{8 2 . 8 6}$ & 0.00 & 1.43 \\
T5_23 & 0.00 & 8.57 & 0.00 & 0.00 & $\mathbf{8 5 . 7 1}$ & 0.00 \\
T6_22 & 0.00 & 0.00 & 1.43 & 8.57 & 1.43 & $\mathbf{9 7 . 1 4}$ \\
No response & 0.00 & 0.00 & 1.43 & 0.00 & 0.00 & 0.00 \\
\hline
\end{tabular}

observed between MBEA global scores and mean rationalized arcsine transformed tone identification scores when both groups were combined $[r(26)=0.56, p=0.002]$ and for the control group [amusics: $r(12)=0.53, p=0.051$; controls: $r(12)=0.71$, $p=0.005]$ : the better the music perception, the better the lexical tone identification. There was also a significant negative correlation between MBEA global scores and mean amplitudes of the third harmonic of FFRs to the speech-in-quiet stimuli (averaged across the six tones) for the control group $[r(12)=-0.63$, $p=0.016$ ): the better the music perception, the smaller the amplitude of the third harmonic of FFRs to the speech-in-quiet stimuli.

Correlation analyses between MBEA global scores and FFR measures of speech-in-noise stimuli revealed significant correlations in several cases for the control group only. First, significant correlations were observed between MBEA global scores and pitch strengths $[r(12)=0.77, p=0.001]$, pitch errors $[r(12)=-0.60$, $p=0.021]$, SNRs $[r(12)=0.66, p=0.010]$, and RMS amplitudes $[r(12)=0.58, p=0.030]$ of FFRs to speech-in-noise stimuli in controls. Second, there was also a significant positive correlation between MBEA global scores and mean amplitudes of the first harmonic of the speech-in-noise stimuli for the control group $[r(12)=0.61, p=0.020]$.

Correlation analyses between MBEA global scores and FFR measures of cello tones revealed significant correlations in several cases for the control group only. First, positive correlations were observed between MBEA global scores and pitch strengths $[r(12)=0.57, p=0.032]$ and SNRs $[r(12)=0.63, p=0.015]$ of FFRs to cello tones in controls. Second, there was also a significant negative correlation between MBEA global scores and mean amplitudes of the third harmonic of the cello stimuli for the control group $[r(12)=-0.81, p<0.001]$.

\section{POWER ANALYSIS}

Power analysis was conducted in order to examine whether the current non-significant group differences in brainstem encoding of speech (in quiet and noise) and musical stimuli were due to sample size or the power of this study. According to Cohen (1988), a study should have at least $80 \%$ power to be worth doing. Suppose we wanted to achieve a medium effect size (Cohen's $d=0.5$ ) with significance level at $p<0.05$, having $n=28$ participants would give us a power of $84 \%$ to find amusics being worse than controls in the tasks. Indeed, the significant group difference in behavioral identification of the six Cantonese tones showed a medium effect size (Cohen's $d=0.508$, on rationalized arcsine transformed scores) in the current study. Therefore, the current finding of intact brainstem encoding of speech (in quiet and noise) and musical stimuli in amusia is unlikely to be inconclusive.

\section{DISCUSSION}

This study investigated the relationship between brainstem representation and behavioral identification of lexical tones as well as brainstem encoding of speech-in-noise and musical stimuli in Cantonese-speaking individuals with congenital amusia, a disorder of pitch processing in music and speech. Measurements of the FFR waveforms revealed no evidence of abnormal brainstem encoding of speech (in quiet and noise) or musical stimuli for amusics relative to controls, in terms of timing, frequency, and amplitude. However, amusics performed significantly worse than controls on identification of lexical tones. The dissociation between brainstem representation and behavioral identification of lexical tones was further confirmed by the lack of correlation between FFR and behavioral pitch and timing measures. No correlation was observed between amusics' music perception scores and FFR measures of speech-in-noise/musical stimuli, either. These findings suggest that amusics' subcortical neural responses simply represent acoustic/sensory properties of the speech or musical stimuli, rather than reflecting their higher-level pitch-processing deficits.

Brainstem responses to speech/music sounds have been considered as biological markers of individuals' auditory, music, and language processing abilities (Chandrasekaran and Kraus, 2010; Skoe and Kraus, 2010). Previous neuroimaging and neurophysiological studies of amusia suggest that amusics' pitch processing deficits may or may not start in the auditory cortex (Albouy et al., 2013a; Peretz, 2013). Given the positive association between the quality of brainstem representation of speech/music sounds and musical expertise (Musacchia et al., 2007; Wong et al., 2007; Parbery-Clark et al., 2009a; Strait et al., 2012), it would be worth exploring whether disordered musical functioning in amusia is related to impaired subcortical representation of pitch-bearing information. Our results revealed no evidence of abnormal brainstem representation of speech (in quiet and noise) or musical stimuli in amusia, across all FFR measures in terms of timing, frequency, and amplitude, suggesting that amusics' pitch processing deficits are unlikely to originate from the auditory brainstem. It has been proposed that the top-down corticofugal pathway may be a potential mechanism for explaining brainstem encoding advantage in tone language speakers and musicians (Chandrasekaran 
and Kraus, 2010). Our current results indicate that if in fact the previous studies can be explained by the corticofugal system, it is not in play in amusics, which suggests that amusics may have a very high level of deficits that are confined within the cortex.

Compared to non-musicians, musicians have been shown to have enhanced brainstem encoding of speech and musical stimuli in a quiet setting (Musacchia et al., 2007). If subcortical encoding of speech/music sounds reflected musical aptitude along the entire spectrum from musicians to non-musician controls and to amusics, we would expect amusics to show impaired brainstem encoding of speech and musical stimuli compared to controls. However, our results indicate normal brainstem encoding of speech and musical stimuli in quiet in Cantonesespeaking amusics, across six different lexical tones and two cello tones (Figures 2-3 and 5; Tables S2 and S4). This suggests that amusics' speech and musical processing deficits are not due to reduced brainstem encoding of speech and musical sounds.

Previous research also suggests that musicians have enhanced brainstem encoding of speech in noise, which is coupled with perceptual enhancement in hearing speech in noise (ParberyClark etal., 2009a; Strait etal., 2012). The strong association between brainstem representation of $\mathrm{F}_{0}$ in noise and perceptual performance on speech-in-noise has also been observed in English-speaking non-musicians (Song et al., 2011). While it is a matter of debate whether musicians indeed have enhanced ability to understand speech in noise compared to non-musicians (Parbery-Clark etal., 2009b; Ruggles et al., 2014), Mandarinspeaking amusics have shown reduced speech intelligibility in both quiet and noise, with natural or flattened $\mathrm{F}_{0}$, relative to normal controls (Liu et al., 2015). Although our current study did not measure participants' behavioral performance on understanding speech in noise, our brainstem data (Figure 4; Table S3) revealed largely normal FFRs to speech in noise in Cantonese-speaking amusics as compared to controls, but with two exceptions. First, controls showed larger first harmonic $\left(\mathrm{F}_{0}\right)$ amplitudes in FFRs to speech in noise than amusics $[F(1,25)=5.11, p=0.033$; Table $\mathrm{S} 3$ ], indicating stronger subcortical spectral encoding of speech in noise in controls relative to amusics. However, amusics demonstrated shorter neural lags than controls in FFRs to Tone 1 in noise $[t(54)=-3.09, p=0.003$; Table S3], suggesting shorter neural conduction time for speech in noise in amusics versus controls. These mixed results, although interesting, may be due to familywise Type I errors (false positives) from multiple comparisons, as no significant group difference was observed in the overall ANOVAs using measures averaged across different tone and noise conditions (Table 2). Further studies are required to examine the relationship between amusics' speech comprehension deficits in quiet and noise and their subcortical and cortical representation of speech in quiet and noise.

Despite demonstrating largely normal brainstem encoding of speech and musical stimuli, a deficit in lexical tone identification was observed for the current sample of amusics. This is consistent with previous findings of impaired lexical tone processing in Mandarin-speaking amusics (Nan et al., 2010; Jiang et al., 2012b; Liu et al., 2012a). For Cantonese, it has been suggested that tones with similar acoustic features, e.g., Tones 2 and 5 (two rising tones),
Tones 3 and 6 (two level tones), and Tones 4 and 6 (two low tones), are in the process of merging into one single category due to the ongoing sound change (Mok and Zuo, 2012; Law et al., 2013; Mok et al., 2013). In our current tone identification task, confusion matrices of amusics and controls suggested that amusics were more likely than controls to confuse between these acoustically similar tones, presumably due to their pitch discrimination difficulty.

In line with previous findings of different performance of amusics on implicit/pre-attentive versus explicit/attentive tasks and conditions (Loui et al., 2008; Peretz et al., 2009; Liu et al., 2010; Hutchins and Peretz, 2012; Mignault Goulet et al., 2012; Omigie et al., 2012; Moreau etal., 2013), the current study observed a dissociation between pre-attentive subcortical representation and perceptual identification of lexical tones in amusia. This suggests that amusics have difficulty mapping tonal patterns onto longtime stored linguistic categories, despite having received sufficient acoustic input of these tones at the brainstem level. This dissociation suggests that amusia is a higher-level pitch-processing disorder. It has been shown that higher-level processing of internal representations of linguistic tones is implicated in the left inferior frontal gyrus (Hsieh et al., 2001). Thus, amusics' tone identification deficits may be related to their structural abnormality in this brain region (Mandell et al., 2007). Further studies are required to examine how amusic tone-language speakers map tonal patterns onto internalized linguistic representations during speech comprehension.

Previous research has led to mixed results regarding the relationship between neurophysiological and behavioral processing of complex sounds. In Bidelman et al. (2011b), Chinese listeners demonstrated musician-like, enhanced brainstem encoding of musical pitch compared to non-musicians, but similar to nonmusicians, they did not achieve musician-level performance on musical pitch discrimination. Similarly, compared to English musicians and non-musicians, Chinese listeners showed larger MMN (mismatch negativity) responses to the within-category difference between their native curvi-linear rising tone and a strictly linear rising pitch, although they were not as accurate as those non-native listeners in discriminating these within-category tones (Chandrasekaran et al., 2009). Nevertheless, several other studies have reported significant correlations between brainstem responses and behavioral measures such as frequency discrimination (Bidelman and Krishnan, 2010; Krishnan et al., 2010a, 2012; Carcagno and Plack, 2011; Marmel et al., 2013) and hearing speech in noise (Parbery-Clark et al., 2009a; Song et al., 2011; Strait et al., 2012). It is possible that the dissociation between neural and behavioral pitch processing in Chinese listeners observed in previous studies was due to the nature of the stimuli used: unfamiliar musical pitches (Bidelman et al., 2011b) and within-category tones (Chandrasekaran et al., 2009), which would naturally lead to inferior performance of Chinese listeners (non-musicians) as compared to musicians and non-native listeners. Using natural and behaviorally relevant lexical tones as stimuli, which were neither unfamiliar nor within-category for native Cantonese listeners, the current study excluded the confounding factor of stimuli but reached similar findings as in previous studies (Chandrasekaran et al., 2009; Bidelman et al., 2011b). Our finding 
of the brain-behavior dissociation in the processing of lexical tones in amusia requires further investigations into the interplay between subcortical and cortical structures along the auditory pathway, as our current results seem to suggest that, although the amusic brainstem maintains detailed representations of lexical tones, it does not lead to perceptual recognition of these tones at the normal level.

A growing number of reports have suggested that FFR is not a direct correlate of the pitch percept itself, but only reflects exogenous stimulus properties, e.g., temporal information originated from the auditory periphery (Bidelman et al., 2011b, 2013; Gockel et al., 2011; Plack et al., 2014). On the other hand, the human "pitch center," which is located in lateral Heschl's gyrus (HG) and anterolateral PT (planum temporale) of the auditory cortex, has been proposed to represent the percept of pitch (Bendor, 2012; Griffiths and Hall, 2012; Plack et al., 2014). Our findings seem to agree with these proposals, as amusics' pitch-processing deficits were not reflected in the brainstem, but were present at the perceptual/behavioral level in acoustic-to-phonetic mapping of lexical tones. A recent study examining categorical perception of vowels also showed that cortical but not brainstem speech representations accounted for the acoustic-to-phonetic mapping of speech sounds (Bidelman et al., 2013).

Finally, it is worth mentioning that the current amusics, being exposed to six lexical tones in Cantonese, which is rare in the world's tone languages (Yip, 2002), may have developed a protective mechanism against brainstem deficits through development, especially in terms of pitch encoding. Although subtle spectral encoding deficits $\left(\mathrm{F}_{0}\right.$ amplitude) for speech in noise were observed for the current Cantonese-speaking amusics (but note amusics' quicker neural conduction time than controls for Tone 1 in noise; Table S3), it is possible that if we examined amusics from non-tonal language backgrounds (e.g., English, French), we would see salient pitch-encoding deficits in the amusic brainstem. However, a recent poster investigating brainstem responses to dissonance in musical stimuli in French-speaking amusics (Cousineau et al., 2014) also observed a trend for normal brainstem encoding of musical dissonance in amusia. Furthermore, a recent psychoacoustic study reported that amusics showed normal spectral and temporal coding of pitch in the auditory periphery (Cousineau etal., 2015). Further studies are required to confirm these observations, and explore how the amusic brainstem encodes more complex musical stimuli compared to normal controls, using participants from both tone and non-tonal language backgrounds.

In summary, the current study revealed a dissociation between subcortical representation (normal) and behavioral identification (impaired) of lexical tones as well as intact brainstem encoding of speech (in quiet and noise) and musical stimuli in Cantonese-speaking individuals with congenital amusia, a disorder of musical and linguistic pitch processing. Future studies are required to investigate how and where along the auditory pathway acoustic features of speech/music sounds start to transform into internalized linguistic/musical percepts in the amusic brain, and how the amusic brainstem encodes more complex musical stimuli compared to normal controls.

\section{ACKNOWLEDGMENTS}

This work was supported by National Science Foundation Grant BCS-1125144, the Liu Che Woo Institute of Innovative Medicine at The Chinese University of Hong Kong, the US National Institutes of Health grants R01DC008333 and R01DC013315, the Research Grants Council of Hong Kong grants 477513 and 14117514, the Health and Medical Research Fund of Hong Kong grant 01120616, and the Global Parent Child Resource Centre Limited. We would like to thank Erika Skoe, Tim Schoof, and Bharath Chandrasekaran for providing us with the Matlab scripts for analyzing FFR data. We also wish to thank Mark Antoniou, Hilda Chan, Antonio Cheung, Judy Kwan, and Doris Lau for their assistance in this research, and two anonymous reviewers for helpful comments.

\section{SUPPLEMENTARY MATERIAL}

The Supplementary Material for this article can be found online at: http://www.frontiersin.org/journal/10.3389/fnhum.2014.01029/ abstract

\section{REFERENCES}

Aiken, S. J., and Picton, T. W. (2008). Envelope and spectral frequencyfollowing responses to vowel sounds. Hear. Res. 245, 35-47. doi: 10.1016/j.heares.2008.08.004

Albouy, P., Mattout, J., Bouet, R., Maby, E., Sanchez, G., Aguera, P.-E., et al. (2013a). Impaired pitch perception and memory in congenital amusia: the deficit starts in the auditory cortex. Brain 136, 1639-1661. doi: 10.1093/brain/ awt082

Albouy, P., Schulze, K., Caclin, A., and Tillmann, B. (2013b). Does tonality boost short-term memory in congenital amusia? Brain Res. 1537, 224-232. doi: 10.1016/j.brainres.2013.09.003

Anderson, S., White-Schwoch, T., Parbery-Clark, A., and Kraus, N. (2013). Reversal of age-related neural timing delays with training. Proc. Natl. Acad. Sci. U.S.A. 110, 4357-4362. doi: 10.1073/pnas.1213555110

Ayotte, J., Peretz, I., and Hyde, K. L. (2002). Congenital amusia: a group study of adults afflicted with a music-specific disorder. Brain J. Neurol. 125, 238-251. doi: 10.1093/brain/awf028

Bendor, D. (2012). Does a pitch center exist in auditory cortex? J. Neurophysiol. 107, 743-746. doi: 10.1152/jn.00804.2011

Bidelman, G. M., Gandour, J. T., and Krishnan, A. (2011a). Cross-domain effects of music and language experience on the representation of pitch in the human auditory brainstem. J. Cogn. Neurosci. 23, 425-434. doi: 10.1162/jocn.2009.21362

Bidelman, G. M., Gandour, J. T., and Krishnan, A. (2011b). Musicians and tonelanguage speakers share enhanced brainstem encoding but not perceptual benefits for musical pitch. Brain Cogn. 77, 1-10. doi: 10.1016/j.bandc.2011.07.006

Bidelman, G. M., and Krishnan, A. (2010). Effects of reverberation on brainstem representation of speech in musicians and non-musicians. Brain Res. 1355, 112 125. doi: 10.1016/j.brainres.2010.07.100

Bidelman, G. M., Moreno, S., and Alain, C. (2013). Tracing the emergence of categorical speech perception in the human auditory system. Neuroimage 79, 201-212. doi: 10.1016/j.neuroimage.2013.04.093

Boersma, P., and Weenink, D. (2001). Praat, a system for doing phonetics by computer. Glot Int. 5, 341-345.

Braun, A., McArdle, J., Jones, J., Nechaev, V., Zalewski, C., Brewer, C., et al. (2008). Tune deafness: processing melodic errors outside of conscious awareness as reflected by components of the auditory ERP. PLoS ONE 3:e2349. doi: 10.1371/journal.pone.0002349

Carcagno, S., and Plack, C. J. (2011). Subcortical plasticity following perceptual learning in a pitch discrimination task. J. Assoc. Res. Otolaryngol. 12, 89-100. doi: 10.1007/s10162-010-0236-231

Chandrasekaran, B., and Kraus, N. (2010). The scalp-recorded brainstem response to speech: neural origins and plasticity. Psychophysiology 47, 236-246. doi: 10.1111/j.1469-8986.2009.00928.x

Chandrasekaran, B., Kraus, N., and Wong, P. C. M. (2012). Human inferior colliculus activity relates to individual differences in spoken language learning. J. Neurophysiol. 107, 1325-1336. doi: 10.1152/jn.00923.2011 
Chandrasekaran, B., Krishnan, A., and Gandour, J. T. (2009). Relative influence of musical and linguistic experience on early cortical processing of pitch contours. Brain Lang. 108, 1-9. doi: 10.1016/j.bandl.2008.02.001

Chao, Y. R. (1930). A system of tone letters. Maître Phon. 45, 24-27.

Chisolm, T. H., Willott, J. F., and Lister, J. J. (2003). The aging auditory system: anatomic and physiologic changes and implications for rehabilitation. Int. J. Audiol. 42, 3-10. doi: 10.3109/14992020309074637

Ciocca, V., and Lui, J. Y. K. (2003). The development of the perception of Cantonese lexical tones. J. Multiling. Commun. Disord. 1, 141-147. doi: 10.1080/1476967031000090971

Cohen, J. (1988). Statistical Power Analysis for the Behavioral Sciences. 2nd Edn. Hillsdale, NJ: Routledge.

Cousineau, M., Bidelman, G. M., Peretz, I., and Lehmann, A. (2014). "Dissonance and the brainstem: insights from natural stimuli and congenital amusia," in The Neurosciences and Music - V: Cognitive Stimulation and Rehabilitation. Dijon: Mariani Foundation.

Cousineau, M., Oxenham, A. J., and Peretz, I. (2015). Congenital amusia: a cognitive disorder limited to resolved harmonics and with no peripheral basis. Neuropsychologia 66, 293-301. doi: 10.1016/j.neuropsychologia.2014.11.031

Drayna, D., Manichaikul, A., de Lange, M., Snieder, H., and Spector, T. (2001). Genetic correlates of musical pitch recognition in humans. Science 291, 1969 1972. doi: 10.1126/science.291.5510.1969

Eggermont, J. J. (2001). Between sound and perception: reviewing the search for a neural code. Hear. Res. 157, 1-42. doi: 10.1016/S0378-5955(01)00259-3

Foxton, J. M., Dean, J. L., Gee, R., Peretz, I., and Griffiths, T. D. (2004). Characterization of deficits in pitch perception underlying "tone deafness." Brain 127, 801-810. doi: 10.1093/brain/awh105

Francis, A. L., and Ciocca, V. (2003). Stimulus presentation order and the perception of lexical tones in Cantonese. J. Acoust. Soc. Am. 114, 1611-1621. doi: $10.1121 / 1.1603231$

Gandour, J., Wong, D., Lowe, M., Dzemidzic, M., Satthamnuwong, N., Tong, Y., et al. (2002). A cross-linguistic FMRI study of spectral and temporal cues underlying phonological processing. J. Cogn. Neurosci. 14, 1076-1087. doi: 10.1162/089892902320474526

Gockel, H. E., Carlyon, R. P., Mehta, A., and Plack, C. J. (2011). The frequency following response (FFR) may reflect pitch-bearing information but is not a direct representation of pitch. J. Assoc. Res. Otolaryngol. 12, 767-782. doi 10.1007/s10162-011-0284-1

Gosselin, N., Jolicoeur, P., and Peretz, I. (2009). Impaired memory for pitch in congenital amusia. Ann. N. Y. Acad. Sci. 1169, 270-272. doi: 10.1111/j.17496632.2009.04762.x

Griffiths, T. D., and Hall, D. A. (2012). Mapping pitch representation in neural ensembles with fMRI. J. Neurosci. 32, 13343-13347. doi: 10.1523/JNEUROSCI.3813-12.2012

Henry, M. J., and McAuley, J. D. (2010). On the prevalence of congenital amusia. Music Percept. 27, 413-418. doi: 10.1525/mp.2010.27.5.413

Henry, M. J., and McAuley, J. D. (2013). Failure to apply signal detection theory to the montreal battery of evaluation of amusia may misdiagnose amusia. Music Percept. Interdiscip. J. 30, 480-496. doi: 10.1525/mp.2013.30.5.480

Hickok, G., and Poeppel, D. (2007). The cortical organization of speech processing. Nat. Rev. Neurosci. 8, 393-402. doi: 10.1038/nrn2113

Holm, S. (1979). A simple sequentially rejective multiple test procedure. Scand. J. Stat. 6, 65-70. doi: 10.2307/4615733

Howell, D. C. (2009). Statistical Methods for Psychology, 7th Edn. Belmont, CA: Cengage Learning.

Hsieh, L., Gandour, J., Wong, D., and Hutchins, G. D. (2001). Functional heterogeneity of inferior frontal gyrus is shaped by linguistic experience. Brain Lang. 76, 227-252. doi: 10.1006/brln.2000.2382

Hutchins, S., Gosselin, N., and Peretz, I. (2010). Identification of changes along a continuum of speech intonation is impaired in congenital amusia. Front. Psychol. 1:236. doi: 10.3389/fpsyg.2010.00236

Hutchins, S., and Peretz, I. (2012). Amusics can imitate what they cannot discriminate. Brain Lang. 123, 234-239. doi: 10.1016/j.bandl.2012.09.011

Hyde, K. L., Lerch, J. P., Zatorre, R. J., Griffiths, T. D., Evans, A. C., and Peretz, I. (2007). Cortical thickness in congenital amusia: when less is better than more. J. Neurosci. 27, 13028-13032. doi: 10.1523/JNEUROSCI.3039-07.2007

Hyde, K. L., and Peretz, I. (2004). Brains that are out of tune but in time. Psychol. Sci. 15, 356-360. doi: 10.1111/j.0956-7976.2004.00683.x
Hyde, K. L., Zatorre, R. J., Griffiths, T. D., Lerch, J. P., and Peretz, I. (2006). Morphometry of the amusic brain: a two-site study. Brain 129, 2562-2570. doi: 10.1093/brain/awl204

Hyde, K. L., Zatorre, R. J., and Peretz, I. (2011). Functional MRI evidence of an abnormal neural network for pitch processing in congenital amusia. Cereb. Cortex 21, 292-299. doi: 10.1093/cercor/bhq094

Jiang, C., Hamm, J. P., Lim, V. K., Kirk, I. J., and Yang, Y. (2010). Processing melodic contour and speech intonation in congenital amusics with Mandarin Chinese. Neuropsychologia 48, 2630-2639. doi: 10.1016/j.neuropsychologia.2010.05.009

Jiang, C., Hamm, J. P., Lim, V. K., Kirk, I. J., and Yang, Y. (2011). Fine-grained pitch discrimination in congenital amusics with Mandarin Chinese. Music Percept. 28, 519-526. doi: 10.1525/mp.2011.28.5.519

Jiang, C., Hamm, J. P., Lim, V. K., Kirk, I. J., Chen, X., and Yang, Y. (2012a). Amusia results in abnormal brain activity following inappropriate intonation during speech comprehension. PLoS ONE 7:e41411. doi: 10.1371/journal.pone. 0041411

Jiang, C., Hamm, J. P., Lim, V. K., Kirk, I. J., and Yang, Y. (2012b). Impaired categorical perception of lexical tones in Mandarin-speaking congenital amusics. Mem. Cognit. 40, 1109-1121. doi: 10.3758/s13421-012-0208-2

Jiang, C., Lim, V. K., Wang, H., and Hamm, J. P. (2013). Difficulties with pitch discrimination influences pitch memory performance: evidence from congenital amusia. PLoS ONE 8:e79216. doi: 10.1371/journal.pone.0079216

Kalmus, H., and Fry, D. B. (1980). On tune deafness (dysmelodia): frequency, development, genetics and musical background. Ann. Hum. Genet. 43, 369-382. doi: 10.1111/j.1469-1809.1980.tb01571.x

Krishnan, A., Bidelman, G. M., and Gandour, J. T. (2010a). Neural representation of pitch salience in the human brainstem revealed by psychophysical and electrophysiological indices. Hear. Res. 268, 60-66. doi: 10.1016/j.heares.2010.04.016

Krishnan, A., Gandour, J. T., and Bidelman, G. M. (2010b). The effects of tone language experience on pitch processing in the brainstem. J. Neurolinguistics 23, 81-95. doi: 10.1016/j.jneuroling.2009.09.001

Krishnan, A., Gandour, J. T., Smalt, C. J., and Bidelman, G. M. (2010c). Language-dependent pitch encoding advantage in the brainstem is not limited to acceleration rates that occur in natural speech. Brain Lang. 114, 193-198. doi: 10.1016/j.bandl.2010.05.004

Krishnan, A., Bidelman, G. M., Smalt, C. J., Ananthakrishnan, S., and Gandour, J. T. (2012). Relationship between brainstem, cortical and behavioral measures relevant to pitch salience in humans. Neuropsychologia 50, 2849-2859. doi: 10.1016/j.neuropsychologia.2012.08.013

Krishnan, A., Xu, Y., Gandour, J., and Cariani, P. (2005). Encoding of pitch in the human brainstem is sensitive to language experience. Brain Res. Cogn. Brain Res. 25, 161-168. doi: 10.1016/j.cogbrainres.2005.05.004

Krizman, J., Marian, V., Shook, A., Skoe, E., and Kraus, N. (2012). Subcortical encoding of sound is enhanced in bilinguals and relates to executive function advantages. Proc. Natl. Acad. Sci. U.S.A. 109, 7877-7881. doi: 10.1073/pnas.1201 575109

Law, S.-P., Fung, R., and Kung, C. (2013). An ERP study of good production visà-vis poor perception of tones in cantonese: implications for top-down speech processing. PLoS ONE 8:e54396. doi: 10.1371/journal.pone.0054396

Liu, F., Jiang, C., Pfordresher, P. Q., Mantell, J. T., Xu, Y., Yang, Y., et al. (2013). Individuals with congenital amusia imitate pitches more accurately in singing than in speaking: implications for music and language processing. Atten. Percept. Psychophys. 75, 1783-1798. doi: 10.3758/s13414-013-0506-1

Liu, F., Jiang, C., Thompson, W. F., Xu, Y., Yang, Y., and Stewart, L. (2012a). The mechanism of speech processing in congenital amusia: evidence from mandarin speakers. PLoS ONE 7:e30374. doi: 10.1371/journal.pone.0030374

Liu, F., Xu, Y., Patel, A. D., Francart, T., and Jiang, C. (2012b). Differential recognition of pitch patterns in discrete and gliding stimuli in congenital amusia: evidence from Mandarin speakers. Brain Cogn. 79, 209-215. doi: 10.1016/j.bandc.2012.03.008

Liu, F., Jiang, C., Wang, B., Xu, Y., and Patel, A. D. (2015). A music perception disorder (congenital amusia) influences speech comprehension. Neuropsychologia 66, 111-118. doi: 10.1016/j.neuropsychologia.2014.11.001

Liu, F., Patel, A. D., Fourcin, A., and Stewart, L. (2010). Intonation processing in congenital amusia: discrimination, identification and imitation. Brain 133, 1682-1693. doi: 10.1093/brain/awq089

Loui, P., Alsop, D., and Schlaug, G. (2009). Tone deafness: a new disconnection syndrome? J. Neurosci. 29, 10215-10220. doi: 10.1523/JNEUROSCI.1701-09.2009 
Loui, P., Guenther, F. H., Mathys, C., and Schlaug, G. (2008). Actionperception mismatch in tone-deafness. Curr. Biol. 18, R331-R332. doi: 10.1016/j.cub.2008.02.045

Mandell, J., Schulze, K., and Schlaug, G. (2007). Congenital amusia: an auditorymotor feedback disorder? Restor. Neurol. Neurosci. 25, 323-334.

Marmel, F., Linley, D., Carlyon, R. P., Gockel, H. E., Hopkins, K., and Plack, C. J. (2013). Subcortical neural synchrony and absolute thresholds predict frequency discrimination independently. J. Assoc. Res. Otolaryngol. 14, 757-766. doi: 10.1007/s10162-013-0402-3.

Mead, R. (1990). The Design of Experiments: Statistical Principles for Practical Applications. Cambridge: Cambridge University Press.

Mignault Goulet, G., Moreau, P., Robitaille, N., and Peretz, I. (2012). Congenital amusia persists in the developing brain after daily music listening. PLoS ONE 7:e36860. doi: 10.1371/journal.pone.0036860

Mok, P. K. P., and Zuo, D. (2012). The separation between music and speech: evidence from the perception of Cantonese tones. J. Acoust. Soc. Am. 132, 27112720. doi: 10.1121/1.4747010

Mok, P. P. K., Zuo, D., and Wong, P. W. Y. (2013). Production and perception of a sound change in progress: Tone merging in Hong Kong Cantonese. Lang. Var. Change 25, 341-370. doi: 10.1017/S0954394513000161

Moreau, P., Jolicoeur, P., and Peretz, I. (2009). Automatic brain responses to pitch changes in congenital amusia. Ann. N. Y. Acad. Sci. 1169, 191-194. doi: 10.1111/j.1749-6632.2009.04775.x

Moreau, P., Jolicæur, P., and Peretz, I. (2013). Pitch discrimination without awareness in congenital amusia: evidence from event-related potentials. Brain Cogn. 81, 337-344. doi: 10.1016/j.bandc.2013.01.004

Musacchia, G., Sams, M., Skoe, E., and Kraus, N. (2007). Musicians have enhanced subcortical auditory and audiovisual processing of speech and music. Proc. Natl. Acad. Sci. U.S.A. 104, 15894-15898. doi: 10.1073/pnas.0701498104

Nan, Y., Sun, Y., and Peretz, I. (2010). Congenital amusia in speakers of a tone language: association with lexical tone agnosia. Brain 133, 2635-2642. doi: 10.1093/brain/awq178

Oldfield, R. C. (1971). The assessment and analysis of handedness: the edinburgh inventory. Neuropsychologia 9, 97-113. doi: 10.1016/0028-3932(71)90067-4

Omigie, D., Pearce, M. T., and Stewart, L. (2012). Tracking of pitch probabilities in congenital amusia. Neuropsychologia 50, 1483-1493. doi: 10.1016/j.neuropsychologia.2012.02.034

Omigie, D., Pearce, M. T., Williamson, V. J., and Stewart, L. (2013). Electrophysiological correlates of melodic processing in congenital amusia. Neuropsychologia 51, 1749-1762. doi: 10.1016/j.neuropsychologia.2013.05.010

Parbery-Clark, A., Skoe, E., and Kraus, N. (2009a). Musical experience limits the degradative effects of background noise on the neural processing of sound. J. Neurosci. 29, 14100-14107. doi: 10.1523/JNEUROSCI.3256-09. 2009

Parbery-Clark, A., Skoe, E., Lam, C., and Kraus, N. (2009b). Musician enhancement for speech-in-noise. Ear Hear. 30, 653-661. doi: 10.1097/AUD.0b013e3181 b412e9

Patel, A. D., Foxton, J. M., and Griffiths, T. D. (2005). Musically tone-deaf individuals have difficulty discriminating intonation contours extracted from speech. Brain Cogn. 59, 310-313. doi: 10.1016/j.bandc.2004.10.003

Patel, A. D., Wong, M., Foxton, J., Lochy, A., and Peretz, I. (2008). Speech intonation perception deficits in musical tone deafness (congenital amusia). Music Percept. 25, 357-368. doi: 10.1525/mp.2008.25.4.357

Peretz, I. (2013). “The biological foundations of music: insights from congenital amusia," in The Psychology of Music, 3rd Edn, ed. D. Deutsch (San Diego, CA: Elsevier), 551-564.

Peretz, I., Ayotte, J., Zatorre, R. J., Mehler, J., Ahad, P., Penhune, V. B., et al. (2002). Congenital amusia: a disorder of fine-grained pitch discrimination. Neuron 33 , 185-191. doi: 10.1016/S0896-6273(01)00580-3

Peretz, I., Brattico, E., Järvenpää, M., and Tervaniemi, M. (2009). The amusic brain: in tune, out of key, and unaware. Brain 132, 1277-1286. doi: 10.1093/brain/awp055

Peretz, I., Brattico, E., and Tervaniemi, M. (2005). Abnormal electrical brain responses to pitch in congenital amusia. Ann. Neurol. 58, 478-482. doi 10.1002/ana.20606

Peretz, I., Champod, A. S., and Hyde, K. L. (2003). Varieties of musical disorders. The Montreal Battery of Evaluation of Amusia. Ann. N. Y. Acad. Sci. 999, 58-75. doi: 10.1196/annals. 1284.006
Peretz, I., Cummings, S., and Dubé, M.-P. (2007). The genetics of congenital amusia (tone deafness): a family-aggregation study. Am. J. Hum. Genet. 81, 582-588. doi: $10.1086 / 521337$

Peretz, I., and Zatorre, R. (2005). Brain organization for music processing. Annu. Rev. Psychol. 56, 89-114. doi: 10.1146/annurev.psych.56.091103.070225

Pinheiro, J. C., and Bates, D. M. (2009). Mixed-Effects Models in S and S-Plus. New York, NY: Springer.

Plack, C. J., Barker, D., and Hall, D. A. (2014). Pitch coding and pitch processing in the human brain. Hear. Res. 307, 53-64. doi: 10.1016/j.heares.2013.07.020

Poeppel, D., and Hickok, G. (2004). Towards a new functional anatomy of language. Cognition 92, 1-12. doi: 10.1016/j.cognition.2003.11.001

Poeppel, D., Idsardi, W. J., and van Wassenhove, V. (2008). Speech perception at the interface of neurobiology and linguistics. Philos. Trans. R. Soc. Lond. B Biol. Sci. 363, 1071-1086. doi: 10.1098/rstb.2007.2160

R Core Team (2014). R: A Language and Environment for Statistical Computing. Vienna: R Foundation for Statistical Computing.

Ruggles, D. R., Freyman, R. L., and Oxenham, A. J. (2014). Influence of musical training on understanding voiced and whispered speech in noise. PLoS ONE 9:e86980. doi: 10.1371/journal.pone.0086980

Russo, N., Nicol, T., Musacchia, G., and Kraus, N. (2004). Brainstem responses to speech syllables. Clin. Neurophysiol. 115, 2021-2030. doi: 10.1016/j.clinph.2004.04.003

Schneider, W., Eschman, A., and Zuccolotto, A. (2002). E-Prime: User's Guide. Pittsburgh, PA: Psychology Software Incorporated.

Skoe, E., and Kraus, N. (2010). Auditory brain stem response to complex sounds: a tutorial. Ear Hear. 31, 302-324. doi: 10.1097/AUD.0b013e3181cdb272

Skoe, E., Nicol, T., and Kraus, N. (2013). The Brainstem Toolbox. Available at: www.brainvolts.northwestern.edu

Smith, J. C., Marsh, J. T., and Brown, W. S. (1975). Far-field recorded frequencyfollowing responses: evidence for the locus of brainstem sources. Electroencephalogr. Clin. Neurophysiol. 39, 465-472. doi: 10.1016/0013-4694(75)90047-4

Sohmer, H., Pratt, H., and Kinarti, R. (1977). Sources of frequency following responses (FFR) in man. Electroencephalogr. Clin. Neurophysiol. 42, 656-664. doi: 10.1016/0013-4694(77)90282-6

Song, J. H., Skoe, E., Banai, K., and Kraus, N. (2011). Perception of speech in noise: neural correlates. J. Cogn. Neurosci. 23, 2268-2279. doi: 10.1162/jocn.2010.21556

Song, J. H., Skoe, E., Wong, P. C. M., and Kraus, N. (2008). Plasticity in the adult human auditory brainstem following short-term linguistic training. J. Cogn. Neurosci. 20, 1892-1902. doi: 10.1162/jocn.2008.20131

Stewart, L., von Kriegstein, K., Warren, J. D., and Griffiths, T. D. (2006). Music and the brain: disorders of musical listening. Brain 129, 2533-2553. doi: 10.1093/brain/awl171

Strait, D. L., Parbery-Clark, A., Hittner, E., and Kraus, N. (2012). Musical training during early childhood enhances the neural encoding of speech in noise. Brain Lang. 123, 191-201. doi: 10.1016/j.bandl.2012.09.001

Studebaker, G. A. (1985). A “rationalized” arcsine transform. J. Speech Hear. Res. 28, 455-462. doi: 10.1044/jshr.2803.455

Thompson, W. F., Marin, M. M., and Stewart, L. (2012). Reduced sensitivity to emotional prosody in congenital amusia rekindles the musical protolanguage hypothesis. Proc. Natl. Acad. Sci. U.S.A. 109, 19027-19032. doi: 10.1073/pnas.1210344109

Tillmann, B., Albouy, P., Caclin, A., and Bigand, E. (2014). Musical familiarity in congenital amusia: evidence from a gating paradigm. Cortex 59, 84-94. doi: 10.1016/j.cortex.2014.07.012

Tillmann, B., Gosselin, N., Bigand, E., and Peretz, I. (2012). Priming paradigm reveals harmonic structure processing in congenital amusia. Cortex 48, 10731078. doi: 10.1016/j.cortex.2012.01.001

Tillmann, B., Nguyen, S., Grimault, N., Gosselin, N., and Peretz, I. (2011a). Congenital amusia (or tone-deafness) interferes with pitch processing in tone languages. Front. Audit. Cogn. Neurosci. 2:120. doi: 10.3389/fpsyg.2011.00120

Tillmann, B., Rusconi, E., Traube, C., Butterworth, B., Umiltà, C., and Peretz, I. (2011b). Fine-grained pitch processing of music and speech in congenital amusia. J. Acoust. Soc. Am. 130, 4089-4096. doi: 10.1121/1.3658447

Tillmann, B., Schulze, K., and Foxton, J. M. (2009). Congenital amusia: a short-term memory deficit for non-verbal, but not verbal sounds. Brain Cogn. 71, 259-264. doi: 10.1016/j.bandc.2009.08.003

Varley, R., and So, L. K. H. (1995). Age effects in tonal comprehension in Cantonese. J. Chin. Linguist. 23, 76-97. 
Vuvan, D. T., Nunes-Silva, M., and Peretz, I. (2014). "Modularity of pitch processing in congenital amusia: a meta-analysis," in The Neurosciences and Music - V: Cognitive Stimulation and Rehabilitation, Dijon: Mariani Foundation.

Williamson, V. J., and Stewart, L. (2010). Memory for pitch in congenital amusia: beyond a fine-grained pitch discrimination problem. Memory 18, 657-669. doi: 10.1080/09658211.2010.501339

Wong, P. C. M., Ciocca, V., Chan, A. H. D., Ha, L. Y. Y., Tan, L.-H., and Peretz, I. (2012). Effects of culture on musical pitch perception. PLoS ONE 7:e33424. doi: 10.1371/journal.pone.0033424

Wong, P. C. M., Skoe, E., Russo, N. M., Dees, T., and Kraus, N. (2007). Musical experience shapes human brainstem encoding of linguistic pitch patterns. Nat. Neurosci. 10, 420-422. doi: 10.1038/ nn1872

Yip, M. (2002). Tone. Cambridge: Cambridge University Press. doi: $10.1017 / \mathrm{CBO} 9781139164559$
Conflict of Interest Statement: The authors declare that the research was conducted in the absence of any commercial or financial relationships that could be construed as a potential conflict of interest.

Received: 25 August 2014; accepted: 06 December 2014; published online: 06 January 2015.

Citation: Liu F, Maggu AR, Lau JCY and Wong PCM (2015) Brainstem encoding of speech and musical stimuli in congenital amusia: evidence from Cantonese speakers. Front. Hum. Neurosci. 8:1029. doi: 10.3389/fnhum.2014.01029

This article was submitted to the journal Frontiers in Human Neuroscience.

Copyright ( $(2015$ Liu, Maggu, Lau and Wong. This is an open-access article distributed under the terms of the Creative Commons Attribution License (CC BY). The use, distribution or reproduction in other forums is permitted, provided the original author(s) or licensor are credited and that the original publication in this journal is cited, in accordance with accepted academic practice. No use, distribution or reproduction is permitted which does not comply with these terms. 